\title{
The formulation for cancer prevention \& therapy
}

\author{
Jerry T. Thornthwaite ${ }^{1,2,3}$, Hare R. Shah ${ }^{1,2}$, Pasupati Shah ${ }^{1,2}$, William C. Peeples ${ }^{1,2}$, Henry Respess ${ }^{1,2}$ \\ ${ }^{1}$ Cancer Research Institute of South Florida, Palmetto Bay, USA \\ ${ }^{2}$ Cancer Research Institute of West Tennessee, Henderson, USA \\ ${ }^{3}$ Freed-Hardeman University, Henderson, USA \\ Email: jtt@criwt.com, hare@criwt.com
}

Received 20 March 2013; revised 2 May 2013; accepted 20 May 2013

Copyright (C) 2013 Jerry T. Thornthwaite et al. This is an open access article distributed under the Creative Commons Attribution License, which permits unrestricted use, distribution, and reproduction in any medium, provided the original work is properly cited.

\section{ABSTRACT}

Our natural product research over nine years has been to discover effective over-the-counter-supplements, designed to facilitate classical treatments in fighting disease, specifically cancer. These supplements are important in antiangiogenic, immune and antioxidant defense systems. The components include Acetyl L-Carnitine (ALC), Alpha Lipoic Acid (ALA), Coenzyme Q10 (CoQ10), Curcumin with Piperine, Genistein, Lentinan, N-Acetylcysteine (NAC), Resveratrol, selenium, Vitamin B Complex, Vitamin C, Vitamin $E$ and zinc. These supplement components are supported for human cancer by over 15,000 references in the scientific literature withover of these being published clinical trials, not including zinc and the vitamins. These chemical defined components from natural origins have demonstrated, either individually or collectively, to have antioxidant, anti-angiogenesis, and immune stimulation properties. Furthermore, the direct cancer cell cytotoxicity for Curcumin, Genistein and NAC have been shown. Some of the antiangiogenesis components that affect the majority if not all pathways of angiogenesis, such as Curcumin, Genistein and NAC, actually stimulate the in vivo production of natural antiangiogenic compounds that include Angiostatin, Endostatin and Thrombospotin 1. All of the components play a role in serving as either water or lipid soluble (able to cross the blood-brain barrier) antioxidants. Curcumin, Genistein, Resveratrol, Lentinan, NAC, zinc, selenium and vitamins $B$ and $C$ all stimulate the immune system. Except for ALC and CoQ10, the other components show anti-inflammatory activity. ALC, Resveratrol along with the $B$ and $C$ vitamins are helpful in treating fatigue. The components that help protect the brain and promote nerve regeneration include ALA, CoQ10, Resveratrol, NAC, selenium, zinc and the $B$ and $C$ vitamins. In conclusion, effec- tive prevention and treatment for diseases such as cancer, heart disease and immune deficiency will require multiple compounds.

Keywords: Cancer Prevention; Formulation

\section{INTRODUCTION}

There is general acceptance that treatment of cancer using surgery with or without radiotherapy remains the first treatment modality for most cancer protocols. Radiotherapy is used quite successfully for many forms of cancer while chemotherapy has become an integral part of a multi-disciplinary treatment of cancers and has served also as a palliative measure in cases of advanced cancer.

Despite advances in the early detection of tumors and in the use of surgery, radiation and chemical therapies for disease management, the worldwide mortality from human cancer remains unacceptably high and has increased in the last few years. In the United States from 1930 through 2004, a trend of increasing cancer deaths is shown among both men and women in Scheme 1 (acs.com 2007). Although advances in the early detection of tumors and in the use of chemotherapy and surgery for disease management have helped to enhance the overall survival of afflicted patients, major improvements in treatments for most human cancers are urgently needed.

Preventing cancer is more important than just treating it. In general, the longer a person lives, the more likely he/she will develop cancer. Prevention will make a huge impact on controlling costs and deaths in coming decades. According to the National Institutes of Health, cancer care in 2010 cost $\$ 263.8$ billion, including direct medical expenses as well as indirect costs due to lost productivity and early death.

Uninsured patients and those from ethnic minorities are substantially more likely to be diagnosed with cancer at a later stage, when treatment can be more extensive 


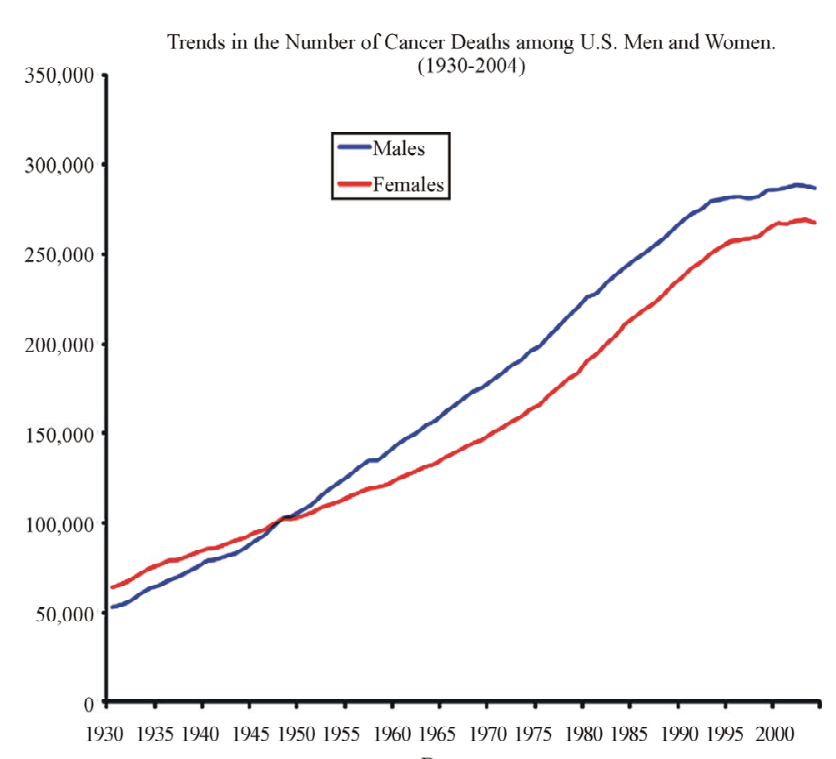

Date

Scheme 1. Cancer death trends.

and more costly. This leads not only to higher medical costs, but also poorer outcomes along with higher cancer death rates. In 2012, about 577,190 Americans are expected to die of cancer. Cancer is the second most common cause of death in the United States, exceeded only by heart disease. Cancer accounts for nearly 1 out of every 4 deaths in the United States (Cancer Facts \& Figures 2012).

The prevention and therapy of cancer may benefit from introduction of new treatments derived from natural products. Many pharmaceutical products approved for human disease treatment are derived from natural sources. The discovery of efficacious compounds for cancer management will benefit from new understanding of the molecular and cellular pathways that regulate tumor proliferation and progression [1].

Patients are becoming acutely aware of the alternative approaches. Nine studies performed worldwide among cancer patients showed that $41.2 \%$ used complementary and alternative medicine during their treatment [2]. In almost all cases of treatment failure, the patient develops distant metastases. While surgery, radiotherapy and chemotherapy are all availa ble to eradicate regional diseases, they are of little value as compared to distant metastases. For such distant metastases, chemotherapy is the recommended approach, but effectiveness is limited by toxic side-effects at high doses and lack of specificity. Furthermore, within the holistic approach of clinical cancer therapy there is now increasing emphasis being given to patient quality of life following these classical treatments, which is encompassed in the term "Hospice". The conclusion is survival should not be the sole criterion for assessing the treatment results. Thus, it has increasingly become an accepted practice for the on- cologist to provide a way to make the patient "comfortable" until they die [3].

It is also well recognized that both radiotherapy and chemotherapy invariably damage or weaken the patient's immunological defenses, which may have already been damaged by the cancer itself. A new awareness has been developed in cancer therapy concerning the importance of the patient's immune system. Biological Response Modifiers (BRMs) have now evolved as the fourth method of cancer treatment in addition to surgery, radiotherapy and chemotherapy. Such treatments with BRMs are considered more biological than directly cytotoxic [4].

In this review paper, we will outline The Formulation which is based on numerous references to key ingredients derived from natural sources in chemically pure form that have direct and safe efficacies in the treatment of cancer. The Formulation can be considered a supplement to the current cancer treatment methods. However, the importance of the components of The Formulation, such as antioxidants, antiangiogenic compounds, natural killer cell and other immune stimulators and direct cancer cytotoxicity, may be considered as a possible first line of treatment and prevention of cancer in the near future.

The Formulation components are supported by over 15,000 references in the scientific literature, not including selenium, zinc and the vitamins with almost all of the components being used in various phase trials as shown in Table 1. To make such a review manageable,

Table 1. Literature references and clinical trials being conducted on each components of the formulation.

\begin{tabular}{|c|c|c|c|}
\hline Formulation & Total Articles & $010-2012$ & Phase Trials \\
\hline Curcumin & 2350 & 576 & $\begin{array}{l}\text { Phase I: } 31,2,3 \text {; } \\
\text { Other: } 22\end{array}$ \\
\hline Genistein & 7332 & 679 & $\begin{array}{l}\text { Phase I: } 14 \text {; } \\
\text { Phase II: 15; Other: } 26\end{array}$ \\
\hline Squalamine & 60 & 4 & $\begin{array}{l}\text { Phase I: } 36,7,8 ; \\
\text { Other: } 6\end{array}$ \\
\hline N-Acetylcysteine & 9196 & 1013 & $\begin{array}{c}\text { Phase I: } 29 \text {, 10; Phase II: } \\
\text { 311, 12, 13; Other: } 79\end{array}$ \\
\hline Arabinoxylan & 319 & 44 & 0 \\
\hline Lentinan & 397 & 8 & $\begin{array}{l}\text { Phase I: 114; Phase II: } 115 \\
\text { Phase III: 116; Other: } 3\end{array}$ \\
\hline Acetyl L-Carnitine & 1304 & 84 & $\begin{array}{l}\text { Phase I: } 317,18,19 \text {; } \\
\text { PhaseIII:120; Other: } 6\end{array}$ \\
\hline Alpha Lipoic Acid & 2455 & 253 & $\begin{array}{c}\text { Phase I: } 2 \text { 21,22; } \\
\text { Phase II:123; Other: } 22\end{array}$ \\
\hline Coenzyme Q10 & 1788 & 192 & $\begin{array}{l}\text { Phase I: } 324,25,26 \text {; } \\
\text { Phase II:127; Other: } 23\end{array}$ \\
\hline Resveratrol & 2287 & 622 & $\begin{array}{l}\text { Phase I: } 128 \text {; } \\
\text { Other: } 7\end{array}$ \\
\hline Selenium & 18740 & 1393 & Phase III: 129 \\
\hline Zinc & 74573 & 5984 & Phase II: 130 \\
\hline
\end{tabular}


we have selected a number of clinical studies based on each component's ability to modify in a positive way, one or more of the attributes of The Formulation.

\section{CURCUMIN}

- Antioxidant

- Antiangiogenic

- Induces apoptosis

- Anti-inflammatory

- Antibacterial

- Antifungal

- Metal chelator

- Cytotoxic to cancer

- Immune enhancing

- Phase I Trials-6

- Phase II Trials-3

As shown in Figure 1, Curcumin (Tumeric) is derived from a spice that comes from the root Curcuma longa, member of the ginger family, Zingaberaceae [4]. It is bright yellow, and has been used as a coloring agent in food in the United States. In India, it has been used for centuries as a spice and as a food preservative and also for its various medicinal properties [5]. Curcumin is one of the most extensively investigated and well-defined chemopreventive phytochemicals [4].

A large number of studies have identified the antioxidant, anti-inflammatory, antiviral, and antifungal properties of curcuminoids [6]. A phase 1 human trial with 25 subjects using up to $8000 \mathrm{mg}$ of curcumin per day for 3 months found no toxicity from curcumin. Five other human trials using $1125-2500 \mathrm{mg}$ of curcumin per day have also found it to be safe. These human studies have found some evidence of anti-inflammatory activity of curcumin. Curcumin has been demonstrated to be safe in six other human trials and has demonstrated anti-inflammatory activity [5].

In phase I clinical studies, curcumin with doses up to 3600 - $8000 \mathrm{mg}$ daily for 4 months did not result in discernable toxicities except mild nausea and diarrhea. The pharmacologically active concentration of curcumin could be achieved in colorectal tissue in patients taking curcumin orally and might also be achievable in tissues

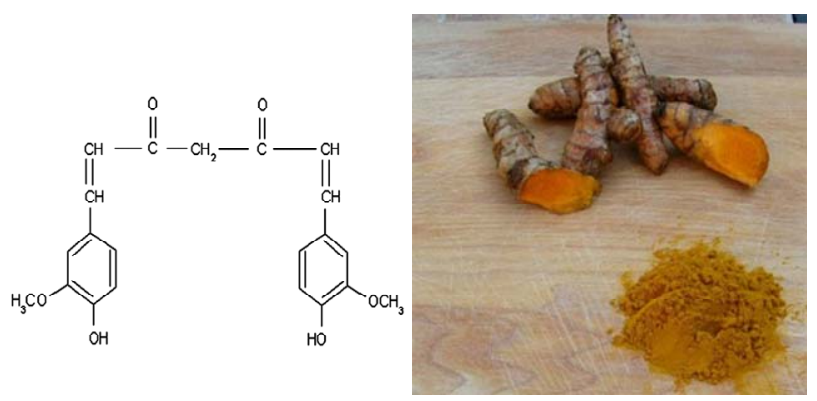

Figure 1. Chemical structure and natural sources of curcumin. such as skin and oral mucosa, which are directly exposed to the drugs applied locally or topically. The effect of curcumin was studied in patients with rheumatoid arthritis, inflammatory eye diseases, inflammatory bowel disease, chronic pancreatitis, psoriasis, hyperlipidemia, and cancers [7]. Curcumin is one of the most extensively investigated and well-defined chemopreventive phytochemicals [8].

The robust activity of curcumin in colorectal cancer has led to five phases I clinical trials being completed showing the safety and tolerability of curcumin in colorectal cancer patients. To date, clinical trials have not identified a maximum tolerated dose of curcumin in humans alongwith doses up to $8000 \mathrm{mg}$ per day. The success of these trials has led to the development of phase II trials that are currently enrolling patients. Overwhelming in vitro evidence and completed clinical trials suggest that curcumin may prove to be useful for the chemoprevention of colon cancer in humans [9].

There is evidence curcumin is a potent immunomodulatory [10]. One of the important factors implicated in chemoresistance and induced chemosensitivity is NF- $\kappa \mathrm{B}$ and curcumin has been shown to down regulate NF- $\kappa \mathrm{B}$ and inhibit $\mathrm{I}-\kappa \mathrm{B}$ kinase thereby suppressing proliferation and inducing apoptosis [11]. It possesses diverse anti-inflammatory and anti-cancer properties following oral or topical administration. It exerts its anti-inflammatory activity by downregulating proinflammatory cytokines such as TNF, IL-1, IL-2, IL-6, IL-8 and IL-12 through possibly the inhibition of the transcription factor, NF- $\kappa \mathrm{B}$ [12] in addition to curcumin's potent antioxidant capacity at neutral and acidic $\mathrm{pH}$; its mechanisms of action include inhibition of several cell signaling pathways at multiple levels, effects on cellular enzymes such as cyclooxygenase and glutathione S-transferases, immunomodulation and effects on angiogenesis and cell-cell adhesion. Curcumin's ability to affect gene transcription and to induce apoptosis in preclinical models is likely to be of particular relevance to cancer chemoprevention and chemotherapy in patients [13].

There are a number of metal chelates with curcumin that show cytotoxicity against cancer cells. Firstly, copper chelates of synthetic curcuminoids showed enhanced antitumor activity. All the compounds were found to be cytotoxic to cultured L929 cells, 50\% inhibition being around $10 \mu \mathrm{g} / \mathrm{ml}$ for curcuminoids and 10 times less for their copper complexes. Copper complex of cinnamyl curcumin which has an extended conjugation showed considerable activity in increasing the life span in $79 \%$ of ascites tumor-bearing mice. Copper chelates of curcuminoids showed a significant reduction $(p<0.001)$ of solid tumor volume in mice [14]. Curcumin possess anticancer and apoptosis-inducing properties in cancer cells. 
A mechanism has been proposed for the cytotoxic action of these compounds against cancer cells that involves mobilization of endogenous copper and the consequent prooxidant action [15]. Furthermore, curcumin acted as a prooxidant causing copper-dependent DNA damage and the induction of apoptosis. Flow cytometry analysis showed that curcumin caused an apoptotic cell death of HL60 cells in a dose- and time-dependent manner. Curcumin can generate reactive oxygen species as a prooxidant in the presence of transition metals in cells, resulting in DNA injuries and apoptotic cell death [16].

Secondly, a novel vanadyl curcumin complex (VO (cur) 2) has been synthesized and its physicochemical property was characterized. VO (cur) 2 was more anticancer or twice as effective as curcumin alone as antiarthritic agent and was more than four times as effective as curcumin alone in inhibiting smooth muscle cell proliferation [17].

Curcumin has been shown to reduce the adenoma burden in patients with colorectal cancer. Curcumin capsules were taken $(3600,1800$, or $450 \mathrm{mg}$ daily) for 7 days. Biopsy samples of normal and malignant colorectal tissue, respectively, were obtained at diagnosis and about 7 hours after the last dose of curcumin. Blood was taken 1 hour after the last dose of curcumin. The results showed that a daily dose of $3.6 \mathrm{~g}$ curcumin achieves pharmacologically efficacious levels in the colorectum with negligible distribution of curcumin outside the gut [18].

Nasopharyngeal carcinoma (NPC) is a common malignant tumor in southern China. A complementary in vitro tumor model showed Curcumin may induce apoptosis and inhibit proliferation of CNE-2Z cells [19].

Furthermore, curcumin can regulate NKC growth of Raji [20] and Ho-8910 cells and induce apoptosis, without significant cytotoxicity to human leukocytes [20].

There is significant experimental antiangiogenic evidence suggesting that curcumin exerts multiple different suppressive effects on MCF-7 human breast carcinoma cells including antiangiogenesis. Curcumin inhibits the transcript levels of 2 major angiogenesis factors VEGF: (vascular endothelial growth factor) and b-FGF (basic fibroblast growth factor) mainly in ER-negative MDAMB-231 breast cancer cells [21]. Nine angiogenesisrelated genes were down-regulated over 5-fold in response to demethoxycurcumin, suggesting that the genetic reprogramming was crucially involved in anti-angiogenesis by this compound [22].

Epidemiological research on prostate cancer risk has identified significant correlations between dietary habits and prostate cancer occurrence. Bemis, et al., 2006 recently reviewed preclinical and clinical data available for dietary agents such as curcumin and describes relevant clinical trials currently being conducted [23].
The published properties of curcumin include anticancer effects in animal model systems, metabolism, biological structure, pharmacokinetics, biological properties implicated in chemoprevention, antioxidant properties, influences on carcinogen-metabolizing enzymes, signal transduction properties and the neoplastic phenoltype, apoptosis evasion, cell proliferation, de-differentiation, migration and invasion, and clinical studies. Campbell and Collett (2005) reviewed curcumin clinical research and summarized the unique properties of curcumin that may be exploited for successful clinical and cancer prevention [24].

In summary, curcumin with or without meal chelates can exhibit a direct cytotoxic, apoptotic effect on cancer cells, while showing antiangiogenic and antioxidant functions. The studies reviewed here provide an insight on the cellular and molecular mechanism(s) by which dietary agents, such as Curcumin, modulate multiple signaling and apoptotic pathways in tumor cells and elucidate their role in both prevention and treatment of cancer [25].

\section{GENISTEIN}

- Antiangiogenic

- Induces apoptosis

- Decreases protein tyrosine kinase

- Decreases topoisomerase II

- Decreases vascular endothelial growth factor

- Decreases prostrate serum albumin

- Increases angiostatin and endostatin

- Increases sensitivity to cisplatin

- Phase I-1

Genistein (4'5,7-trihydroxyisoflavone) occurs as a glycoside (genistin) in the plant family Leguminosae, which includes the soybean (Glycine max). The chemical structure of genistein is a diphenolic structure as shown below in Figure 2.

As of 2012, there are over 4500 genistein studies in peer-reviewed primary publications, almost 900 pertain to its antitumor capabilities and more than 400 describe its mechanism of action in normal and malignant human and animal cells, animal models, in vitro experiments, or phase I/II clinical trials.



Figure 2. Chemical structure and natural source of genistein. 
Epidemiological studies suggest that genistein may reduce the risk of tumor formation [26]. The mechanisms of action include the inhibition of protein tyrosine kinase (PTK), the inhibition of topoisomerase II, the down regulation of the expression of about 11 genes, including VEGF. Genistein can also inhibit the expression of gangliosides and other carbohydrate antigens that can mask immune recognition. Genistein works synergistically with tamoxifen, cisplatin, 1,3-bis2-chloroethyl-1-nitrosurea, dexamethasone, daunorubicin and tiazofurin, and bioflavanoid food supplements such as quercetin, greentea catechin, and black-tea arubigins. Genistein increases melanin production to protect melanocytes of the skin of Caucasians from UV-B radiation-induced melanoma [27].

Genistein is believed to have the potential to lower the incidence of metastatic prostate cancer. Genistein is shown to have kinase inhibitory effects in vivo. The specific suppression of focal adhesion kinase activity was shown to precede induction of apoptosis [28]. The National Cancer Institute is examining genistein as an oral chemotherapeutic for prostate cancer. Those on a Western diet typically have low levels of blood isoflavones. Mean concerntration of plasma/serum genistein were $5.7 \mathrm{nmol} / \mathrm{L}$ in an American study, while the concentration in a Japanese study was $248 \mathrm{nmol} / \mathrm{L}$ (range, 90 to $1204 \mathrm{nmol} / \mathrm{L}$ ). With soy supplementation excretion halflives for genistein is seven hours [27]. Lazarevic, et al. (2012) support genistein as a chemopreventive agent in prostate cancer [29]. Genistein at a dose that can be easily obtained from a diet rich in soy reduced the level of serum PSA in patients with localized prostate cancer, without any effects on hormones. It was well tolerated and had a beneficial effect on blood cholesterol [30].

Genistein has been reported to be a natural chemopreventive in several types of human cancer, being shown to induce cell cycle arrest and apoptosis of bladder cells. Among isoflavones tested, genistein has been proven to be the most potent inhibitor of angiogenesis in vitro and in vivo. Genistein exhibited a dose-dependent inhibition of expression and excretion of vascular endothelial growth factor and platelet-derived growth factor [26].

A mixture of isoflavones produces a synergistic effect that causes even greater anti-tumorigenic effects than any single compound with the values of most cancer cell lines $(3-5 \mu \mathrm{g} / \mathrm{ml}$ or $7.9 \mu \mathrm{M})$ within the reach of isoflavones. Most significantly, genistein has been shown to be preventative for human urinary tract infection. Genistein does not exhibit toxicity to normal bladder cells with the normal physiological range of urine excretion $(10 \mu \mathrm{g} / \mathrm{ml})$. Anti-angiogenesis is one of the most important mechanisms in explaining how soy isoflavones are anti-cancerous [26].

The resistance of renal cell carcinoma (RCC) to tra- ditional therapies or systemic therapies, where only a small percentage of patients actually benefit from immunotherapy with INF and IL-2, leave few options that may be effective. Genistein has been identified as a viable treatment option. Genistein has increasingly been found to treat cancer by a multidimensional approach [26-28,31].

Protein tyrosine kinases (PTKs) play an important role in cell growth. PTKs are associated with cell receptors for EGF, platelet-derived growth factor (PDGF), insulin and insulin-like growth factors (IGF), suggesting that tyrosine phosphorylation plays an important role in cell proliferation and transformation [27].

Topoisomerases introduce transient breaks in DNA. They participate in DNA replication, transcription, integration, and transposition and are also related to transformation by ras-oncogenes. Genistein inhibits the formation of a covalent complex between topoisomerase II and DNA and suppressed the growth the transformed cells [27].

At the cellular level, genistein inhibits cell proliferation, induces apoptosis, induces differentiation, and modulates cell cycle progression. At the molecular level, genistein inhibits the activity of protein tyrosine kinase, topoisomerase II, aromatase, and $17 \beta$-hydroxysteroid oxidoreductase [31].

Genistein is antiangiogenic in vitro and in vivo. Genistein has strong inhibitory effects on the expression of VEGF mRNA and bFGF in RCC cell lines in vitro. VEGF and bFGF are the main angiogenic factors in RCC, so genistein may be antiangiogenic in vitro, but the effect is unknown in vivo. Genistein has been shown to have an inhibitory effect on cell proliferation in leukemia, neuroblastoma, rhabdomyosarcoma, prostate cancer, and bladder cancer [31].

Human cancer cell experiments show that genistein can induce apoptosis by: fragmentation of DNA; activation of caspase-3 (CPP32b); cleavage of poly (ADPribose) polymerase (PARP); downregulation of $\mathrm{Bcl}-2$ (apoptosis inhibitor); enhancement of Bax protein (antagonizes the anti-apoptotic function of Bcl-2); increase of Bax:Bcl-2 ratio; induction of p21WAF1, which downregulates cyclin $\mathrm{B}$ and thereby arrests the cell cycle at the G2/M phase and promotes apoptosis by p53-independent pathway and causes inhibition of the activation of NK$\kappa \mathrm{B}[27]$.

Genistein is considered to enhance the cytotoxicity of radiation. In Reuber H35 hepatoma cells, survival was reduced by a factor of 20 with irradiation alone and by a factor of ten thousand when radiation was administered in the presence of genistein. Based on similar findings in prostate cancer, Hillman et al. 2001 recommended a potential combination of genistein with radiation for the treatment of prostate cancer [32]. The radiation enhan- 
cement is attributed to inhibition of topoisomerase II activity, which is involved in replication, transcription and probably DNA repair [32].

De la Taille et al. (2001) reported that daily intake of 40 grams of soybeans may significantly lower serum levels of prostate-specific antigen (PSA) [33]. Ghafar et al. (2002) reported that 44 days of treatment with genistein plus a polysaccharide from Basidiomycetes reduced serum PSA levels by $4.2 \mathrm{ng} / \mathrm{mL}$ from a pretreatment level, and genistein can decrease PSA mRNA [34-37]. Serum PSA appears to be a useful measure of genistein's efficacy alone or in combination with irradiation or chemotherapeutic drugs for prostate cancer [27].

Although genistein has many potentially therapeutic actions against cancer, its biphasic bioactivity (inhibitory at high concentrations and activating at low concentrations) requires caution in determining therapeutic doses of genistein alone or in combination with chemotherapy, radiation therapy, and/or immunotherapy [27].

Genistein showed an up-regulation of angiogenesis inhibitors-plasminogen activated inhibitor-I, endostatin, angiostatin, and thrombospondin-1. Endostatin and angiostatin are novel molecular targets of genistein. Investigations show more evidence that soy-based foods are natural dietary supplements promoting the inhibition of tumor angiogenesis [26].

Endostatin has a direct anticancer action through blocking the activation of MMP-2, -9 , and -13 , in tumor cells. Angiostatin significantly inhibits the growth and MVD of human bladder cancer in SCID mice. Genistein upregulates endostatin and angiostatin to provide novel mechanisms for isoflavones to reverse the angiogenic switch of epithelial cancer. Isoflavones also suppress the growth and DNA synthesis of endothelial cells in vitro. Some of the biochemical targets of soy isoflavones that are over-expressed in endothelial tumor tissue include TF, VEGF, PDGF and MMP-2. Soy isoflavones are believed to have a combination of sup- pression effects on tumor cells [26].

Angiogenesis is presently one of the most powerful strategies for treating cancer, and endothelial cells play a pivotal role in the process of angiogenesis [38]. Tumor angiogenesis is necessary for the progression of human cancer [39]. Therapeutic angiogenic inhibitors are designed to either impede the pathogenesis of tumor angiogenesis or to destroy the present vasculation of the tumor [26]. Several in vitro studies document the inhibition of angiogenesis by genistein. Shao, et al., (1998) showed that genistein decreased vessel density and the production and release of vascular endothelial growth factor (VEGF) and TGF- $\beta 1$ [40]. Li and Sarkar (2002a, 2002b) have shown that genistein downregulated 11 genes including VEGF, IN U87 and HT1080 renal carcinoma cells [27,41-43]. Genistein, a tyrosine kinase inhi- bitor, is known to inhibit both tumor growth and angiogenesis. The precise molecular mechanism(s) by which genistein affects endothelial cells was investigated using cDNA microarrays. There were 256 genes of human umbilical vein endothelial cells (HUVECs) affected by 10 microM genistein that showed an altered expression of more than two fold. Among them were the genes related to cell proliferation, adhesion, transcription, translation, metabolism, cytoskeleton, apoptosis, kinases, and functionally unknown. Genistein affects endothelial cells as a negative mediator of proliferation and angiogenesis in vitro, partially by down-regulating cell adhesion-related genes and impairing cell adhesion [38].

The incidence of hormone-related diseases such as prostatic, breast, ovarian, and endometrial cancer is lower in Asian populations compared to Western countries. Genistein is postulated to be responsible for the lower incidence of hormone-related disease. Konstan-takopoulos, et al. (2006) showed at physiological concentrations, genistein is able to elicit pleiotropic effects on a variety of pathways believed to be involved in tumorigenesis [44].

Genistein enhances antitumor activities of several chemotherapeutic agents. Genistein increases the antiproliferative effect of cisplatin 1.3 fold in HTB-186 medulloblastoma cell line [45]. Raynal, et al. (2008) evaluated the in vitro and in vivo antileukemic activity of genistein [46]. They observed that it produced a doseand time-dependent antineoplastic activity against myeloid and lymphoid leukemic cell lines. Genistein treatment of the leukemic cells reactivated tumor suppressor genes that were silenced by aberrant DNA methylation. Due to the longer half-life of genistein in humans, a soy-enriched diet has the potential to produce plasma levels of this isoflavone in the range of concentration in vitro that produced an antileukemic activity [46]. There is strong molecular in vivo evidence in support of our hypothesis that inactivation of the NF- $\kappa \mathrm{B}$ signaling pathway by genistein results in the chemo sensitization of pancreatic tumors to cisplatin [47].

Other in vitro studies have reported that the effect of genistein is enhanced by polyphenol food supplements including: curcumin, epigalloeicatechin, EGCG (greentea derived) and thearubigin (black-tea derived), and by mineral such as vanadium.

Genistein in combination with green-tea polyphenol EGCG induced apoptosis and enhanced p53 immunoreactivity in the 184-b5 breast cancer cell line [27]. Dijkstra et al. (2010) states that higher intakes of soy foods and fatty fish may lower the risk of fibroadenomas [48].

The development of cancer is associated with disorders in the regulation of the cell cycle with known sequence of events that regulate cell cycle progression 
[49] including protein kinase complexes composed of cyclin and cyclin-dependent kinase (CDK) molecules. The cyclins are CDK binding partners which are required for kinase activity and their protein levels are intimately linked to the cell cycle stage. Dietary agents identified from fruits and vegetables, such as curcumin, resveratrol, and genistein, can act to modulate the effects of deregulated cell cycle check points [50].

Cancer prevention strategies making use of combined agents with distinct molecular mechanisms, rather than individual agents, are considered promising for higher efficacy and lower toxicity [51].

Genistein also appears to have prophylactic value [52]. There is a reduced risk of cancer among Japanese and Finnish populations that have high consumption of genistein. Genistein as a food supplement can be given to women from prepubertal stage of life so that it would be beneficial in arresting tumor initiation. Genistein may avoid the risk of developing cancer in both men and women who have risk factors for gender-based cancers, such as familial expression of BRCA 1 and 2 [27]. Sarkar, et al. (2006) showed results that suggested genistein and synthetic structurally-modified derivatives of isoflavone may be promising agents for cancer chemoprevention and therapy either alone or in combination with existing chemotherapeutic agents [53].

Perabo, et al. (2008) has described the difficulty in making definite statements or conclusions on clinical efficacy of genistein because of the great variability and differences of the study designs, small patient numbers, short treatment duration and lack of a standardized drug formulation [54]. Nagata, et al. (2007) examined associations between nutritional and other lifestyle factors and the prevalence of prostate cancer in a case-control study of Japanese men [55]. Two hundred patients and 200 age-matched controls (+/-5y) were selected from 3 geographic areas of Japan. Their findings indicated that isoflavones might be an effective dietary protective factor against prostate cancer in Japanese men. Also, soy foods and enterolactone metabolized from dietary lignans protect against prostate cancer in older Scottish men [56]. Furthermore, Kurahashi, et al. (2007) found that isoflavone intake was associated with a decreased risk of localized prostate cancer $(n=43,509)$ [57]. High serum concentrations of isoflavones were associated with a decreased risk for gastric cancer [58].

In summary, these reports indicate that genistein exhibits strong, direct anticancer and antiangiogenic activity. The biological effects of genistein are the inhibition of tyrosine kinases and the inhibition of hypoxic activation of hypoxia-inducible factor-1 (HIF-1), one of the main regulators in the inhibition of VEGF and other angiogenic gene expression.

\section{SQUALAMINE}

- Antiangiogenic

- Synergistic with carboplatin and paclitaxel

- Decreases vascular endothelial growth factor

- Decreases basic fibroblast growth factor

- Decreases platelet derived growth factor

Cartilage is a natural source of material with strong antiangiogenic activity [59]. Clinical information on shark cartilage and drugs such as neovastat and squalamine has been demonstrated. Because their entire endoskeleton is composed of cartilage, sharks are thought to be an ideal source of angiogenic and tumor growth inhibitors. Shark cartilage extract has shown antiangiogenic and antitumor activities in animals and humans. The oral administration of cartilage extract was efficacious in reducing angiogenesis. Squalamine, a low molecular weight aminosterol and the anticancer component of shark cartilage, showed strong antitumor activity when combined with chemotherapeutic materials. The structure is shown in Figure 3.

Squalamine is an antiangiogenic molecule with a unique mechanism of action that blocks endothelial (blood vessel) cell activation, migration and proliferation by multiple growth factors. Squalamine's intracellular blockade of multiple growth factors contrasts with many other angiogenesis inhibition programs that only affect one or two pathways of angiogenesis [60]. The angiogenic tissue inhibitor of metalloprotease 3 (TIMP-3) and tumor suppressor protein (snm23) genes from shark cartilage were cloned and characterized [59].

Squalamine has demonstrated antiangiogenic properties in multiple clinical trials, both as a single-agent and in combination with standard chemotherapy. Squalamine blocks the action of a number of angiogenic growth factors, including vascular endothelial growth factor

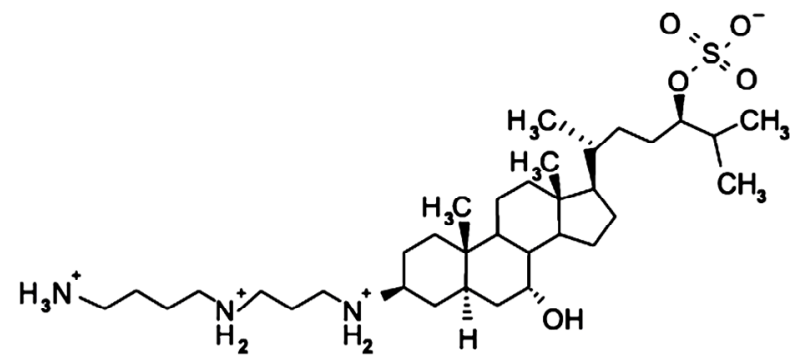

(a)

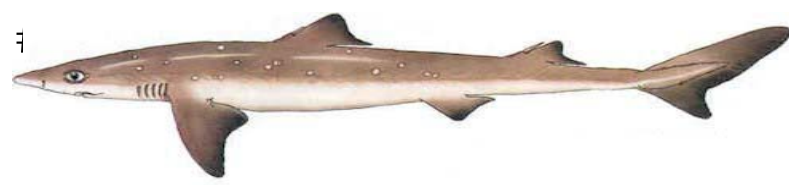

(b)

Figure 3. (a) Chemical structure of squalamine; (b) Natural source of squalamine (dog fish shark). 
(VEGF). The mechanism of action is due to the specific entry of squalamine into activated endothelial cells through membrane invaginations known as caveolae. This unique mechanism has three principal anti-angiogenic effects on endothelial cells: 1) blockage of cell signals from multiple growth factors including VEGF and bFGF, altering cellular activation and cell division; 2) decreased expression of surface integrin alpha-v-beta-3, altering cell-cell interactions; and 3) altered cytoskeletal structure, decreasing motility [59].

A Phase I study of squalamine, a novel antiangiogenic agent originally isolated from the dogfish shark Squalus acanthias, was conducted in patients with advanced cancers to: 1) determine the maximum tolerated dose (MTD), dose-limiting toxicity (DLT) and pharmacokinetics of squalamine lactate when given as a 120 -h continuous IV infusion every two weeks; and 2) to obtain information on prolonged $(>120-\mathrm{h})$ continuous IV infusions in patients who have tolerated $120-\mathrm{h}$ infusions. Preclinical evidence of synergy with cytotoxic agents and demonstration of human safety from this trial have shown efficacy in patients with late stage lung cancer and ovarian cancer [61].

The phase IIa trial in non-small cell lung cancer was designed to exam the preliminary efficacy and safety of Squalamine and combined with the standard chemotherapeutic agents carboplatin and paclitaxel. In patients with Stage IIB or Stage IV advanced disease objective responses (about $500 \mathrm{mg}$ /day based on a $160 \mathrm{lb}$. male or $120 \mathrm{lb}$. female) were observed in $36 \%$ of patients receiving $300 \mathrm{mg} / \mathrm{m}^{2} /$ day for one or more cycles. $31 \%$ of patients (11 of 36) experienced an objective response. An objective response was seen as $50 \%$ or greater reduction in tumor size [60].

A phase I/IIA study was designed to assess the safety, clinical response, and pharmacokinetics of squalamine when administered as a 5-day continuous infusion in conjunction with standard chemotherapy every 3 weeks in patients with stage IIIB (pleural effusion) or stage IV non-small cell lung cancer. The starting dose of squalamine was $100 \mathrm{mg} / \mathrm{m}^{2} /$ day and escalated to $400 \mathrm{mg} / \mathrm{m}^{2} /$ day; two of three patients at $400 \mathrm{mg} / \mathrm{m}^{2} /$ day had doselimiting toxicity that included grade $3 / 4$ arthralgia, myalgia, and neutropenia. The combination of squalamine given daily for 5 days, with paclitaxel and carboplatin given on day 1 , is well tolerated. Patient survival data and the safety profile of this drug combination suggests that the use of squalamine given at its maximum to-lerated dose with cytotoxic chemotherapy should be explored further as a potentially effective therapeutic strategy for patients with stage IIIB or IV non-small cell lung cancer [62].

At the recommended Phase II dose of $500 \mathrm{mg} / \mathrm{m}^{2} /$ day, squalamine is well tolerated and results in plasma con- centrations at least an order of magnitude higher than those required for prominent antiangiogenic effects in preclinical studies [63].

Gateways to clinical trials, a guide to the most recent clinical trials in current literature [64], shows squalamine has been granted Orphan Drug designation for the treatment of ovarian cancer by the U.S. Food and Drug Administration (FDA) five years ago [65]. Squalamine has been found in a therapeutic clinical trial to have positive results against non-small cell lung cancer (NSCLC). Angiogenesis resulting from age-related macular degeneration (AMD) is the leading cause of legal blindness among adults age 50 or older in the Western world. About 25 - 30 million people are affected globally with this number expected to triple over the next 25 years. VEGF-A therapy has revolutionized the treatment [65]. The drug binds to a "chaperones" calmodulin to a intracellular compartment and blocks angiogenesis at several levels [66]. VEGF-A has been implicated in recent years as the major factor responsible for neovascular and exudiative disease of the eye [67]. AMD appears to come in two types: the "dry" form and the more severe "wet" form. Dry AMD, the more common and milder form of AMD, accounts for $85 \%$ to $90 \%$ of all cases. Dry AMD results in varying forms of sight loss and may or may not eventually develop into the wet form. Although the wet form of AMD accounts for only 10 - 15 percent of all $\mathrm{AMD}$, the chance for severe sight loss is greater. It is responsible for 90 percent of severe vision loss associated with AMD.

In North America alone, approximately 200,000 new cases of wet AMD are diagnosed each year. Wet AMD is caused by the growth of abnormal blood vessels, choroidal neovascularization (CNV), under the central part of the retina of the macula [65].

Squalamine shows strong anti-angiogenic activity in vitro alongwith other naturally occurring antiangiogenic compounds shows great promise in treating AMD. The primary actions include blockade of mitogen-induced actin polymerization, cell-cell adhesion and cell migration, leading to suppression of endothelial cell proliferation. Preclinical studies have demonstrated that systemic squalamine administration in primates leads to inhibition of the development of ocular neovasculation and partial regression of new vessels [65]. The dose for squalamine to produce these effects is $12 \mathrm{mg} / \mathrm{m}^{2}$ twice weekly, which is less than $10 \%$ of the doses currently being used successfully in squalamine clinical trials for patients with advanced cancers [62].

Squalamine was found to exhibit little systemic toxicity and was generally well tolerated by treated patients with various solid tumor malignancies, including ovarian, non-small cell lung and breast cancers [1].

Xenograft tumor shrinkage was seen for the MV-522 
tumor in combination treatments including Squalamine; whereas, no tumor shrinkage was seen when squalamine was omitted from the treatment regimen. Squalamine treatment was found to retard two cellular events necessary for angiogenesis, inducing disorganization of Factin stress fibers and causing a concomitant reduction of detectable cell the surface molecular endothelial cadherin (VE-cadherin). We propose that the augmentation by squalamine of cytotoxicity from platinum-based therapies is attributable to interference by squalamine with the ability of stimuli to promote endothelial cell movement and cell-cell communication necessary for growth of new blood vessels in xenografts after chemotherapeutic injury to the tumor [60].

Several classes of agents that now exist target the different steps involved in angiogenesis. Drugs such as squalamine, celecoxib, ZD6126, TNP-470 and those targeting the integrins are also being evaluated in lung cancer [68].

Squalamine is a natural antiangiogenic sterol, and its potential role in treatment of ovarian cancers with or without standard cisplatin chemotherapy was assessed. Since HER-2 gene overexpression is associated with cisplatin resistance in vitro and promotion of tumor angiogenesis in vivo, the response of ovarian cancer cells with or without HER-2 gene overexpression to squalamine and cisplatin was evaluated both in tumor xenograft models and in tissue culture. In in vitro studies, we found that squalamine does not directly affect proliferation of ovarian cells. However, squalamine significantly blocked VEGF-induced activation of MAP kinase and cell proliferation in human vascular endothelial cells [69].

The progressive growth and spread of many solid tumors depends, in part, on the formation of an adequate blood supply and tumor angiogenesis has been reported to have prognostic significance in several human cancers. Therapy directed toward the vasculature of solid tumors is now being pursued as an important new direction in cancer treatment because avascular tumors exhibit only limited growth and tumor aggressiveness, and metastatic potential commonly correlates with tumor vascularity. Vascular endothelial growth factor (VEGF) is produced by most solid tumors and elicits a mitogenic effect on tumor-associated endothelial cells. VEGF binding to receptor tyrosine kinases triggers activation of downstream signaling enzymes, including MAP kinases, which in turn, regulate gene expression and specific endothelial cell responses including proliferation, migration and apoptosis. Several studies have suggested that VEGF plays an important role in the progression of many cancers. Growth factor pathways, such as those dependent on EGF and HER-2 receptors, appear to upregulate VEGF production in solid tumors. Since EGF and HER family receptors are activated and/or overexpressed in significant numbers of human cancers, these growth factor receptor pathways may play a role in promoting further growth of human malignancy by increasing VEGFdependent tumor angiogenesis $[68,69]$.

Akhter, et al. (1999) proposed one mechanism of action of squalamine to involve inhibition of the mammalian brush-border $\mathrm{Na}^{+} / \mathrm{H}^{+}$exchanger isoform NHE3 [70]. The $\mathrm{Na}^{+} / \mathrm{H}^{+}$exchanger is a transport protein that is known to regulate changes in cell volume or cell shape.

Squalamine was found to inhibit rat brain endothelial cell proliferation and migration induced by mitogens, such as VEGF, bFGF, Platelet Derived Growth Factor (PDGF) and scatter factor/hepatocyte growth factor. In the absence of these mitogens, squalamine was found to have no direct effect on survival or proliferation of endothelial cells. In addition, squalamine was also found to inhibit proton secretion by mitogenstimulated endothelial cells, a finding consistent with results reported by Akhter, et al. [70] An interesting finding of this study involved the direct application of squalamine to 4-day-old chick embryo vasculature. After only $20 \mathrm{~min}$, squalamine elicited constriction of the smallest capillaries throughout the yolk sac, with entrapment of red cells. This acute remodeling process resulted in narrowed vascular segments and blocked erythrocyte movement and was confirmed by histological examination of treated and untreated yolk sacs. Since these new vessels are composed solely of endothelial cells, the luminal narrowing was concluded to be due to squalamine-induced changes in the shape or volume of endothelial cells. Immunohistochemical analyses of these tumors after treatment with squalamine revealed significant reductions in tumorassociated blood-vessel density.

$\mathrm{Li}$, et al. conducted studies of human ovarian tumorassociated angiogenesis. Ovarian cells were found to secrete significant levels of VEGF, a direct activator of angiogenesis, but squalamine did not reduce VEGF secretion by tumor cells, and it evoked no direct growth inhibition of ovarian cells in vitro [69]. However, squalamine at doses as low as $160 \mathrm{nM}$ did halt the proliferation of human vascular endothelial cells and markedly reduced VEGF-induced capillary tube-like formations by vascular endothelial cells growing in Matrigel culture. Squalamine interference with these downstream signaling pathways in vascular endothelial cells may be critical in disrupting the process of tumor-associated angiogenesis [70].

Studies by Teicher, et al. [71] noted that squalamine as a single agent has a modest effect on tumor growth delay on rat 13,762 mammary carcinoma, with squalamine dosing at $40 \mathrm{mg} / \mathrm{kg}$ [71]. Moreover, it was found that the number of lung metastases decreased when mice were treated with squalamine. Specifically, by day 20 , the 
numbers of metastases were reduced to half of those present in controls. Since lung metastases are actively implanting and growing using new blood vessels, this effect of squalamine suggests that it has strong antiangiogenic potency [1].

Previous studies have suggested that VEGF plays an important role in progression of ovarian cancer. Ovarian cancer is the most deadly gynecologic malignancy. Although advances in chemotherapy and surgery have helped to improve the overall survival of afflicted patients, 5-year survival rates from ovarian cancer remained about $44 \%$ in the early part of this decade. By the time many patients are diagnosed with ovarian cancer, peritoneal dissemination of the tumor has often occurred. This growth and spread of ovarian cancers depends, in part, on formation of an adequate blood supply. Tumorassociated angiogenesis is essential for growth of most solid tumors, and neovascularization has also been shown to have prognostic significance in epithelial ovarian cancer [72-74].

Administration of squalamine in combination with cisplatin led to enhanced levels of apoptosis in several ovarian tumor cells assessed in vivo [72].

On the basis of strong evidence of antiangiogenic and antitumor properties of squalamine, it was selected for clinical development as a therapeutic agent for treatment of human malignancies. The investigators recruited 19 patients with an Eastern Cooperative Oncology Group (ECOG) performance status of 2 with advanced nonleukemic cancers. Squalamine was administered as a continuous intravenous infusion over $120 \mathrm{~h}$, with repeat dosing every 14 days. The best-tolerated dose of squalamine was found to be $192 \mathrm{mg} / \mathrm{m}^{2} /$ day, although a dose of $384 \mathrm{mg} / \mathrm{m}^{2} /$ day also appeared to be well-tolerated in patients without prior exposure to squalamine [73].

Natural products have served to provide a basis for many of the pharmaceutical agents in current use in cancer therapy and prevention. Squalamine, a natural steroidal compound, causes changes in vascular endothelial cell shape and has been reported to possess significant antiangiogenic activity in models of lung, breast, brain and ovarian cancer. In addition, studies using Lewis lung carcinoma found that the number of metastasis was reduced by half after treatment, which confirms the antiangiogenic potency of squalamine. Squalamine exhibited little systemic toxicity in Phase I-II clinical trials and is well tolerated by treated cancer patients [74].

Since HER-2 gene overexpression is associated with cisplatin resistance in vitro and promotion of tumor angiogenesis in vivo, the response of ovarian cancer cells with or without HER-2 gene overexpression to squalamine and cisplatin was evaluated both in tumor xenograft models and in tissue culture. Profound growth inhibition was elicited by squalamine alone and by combined treatment with squalamine and cisplatin for both parental and HER-2-overexpressing ovarian tumor xenografts. Vascular endothelial growth factor (VEGF) is produced by most solid tumors and elicits a mitogenic effect on tumor-associated endothelial cells, and several studies suggest that VEGF plays an important role in progression of ovarian cancer [69,72-74].

In summary, squalamine has shown to be useful for the treatment of important diseases such as cancers (lung, ovarian, brain, and others), age-related macular degeneration (AMD) and the control of body weight in man [75]. Squalamine causes changes in vascular endothelial cell shape and has been reported to possess significant antiangiogenic activity. Squalamine is somewhat unique among most current anti-angiogenic agents in development because it inhibits endothelial cell proliferation and migration induced by a wide variety of growth factors, including basic Fibroblast Growth Factor (bFGF) and VEGF. This broad antiangiogenic activity of squalamine may result from its inhibition of surface sodium proton exchangers (thus altering intracellular $\mathrm{pH}$ and thereby impeding intracellular signaling by several growth factors) and other downstream signaling pathways in endothelial cells [75].

\section{N-ACETYL CYSTEINE (NAC)}

- Antioxidant

- Antiangiogenic

- Treats Tylenol overdose

Antioxidants have been heralded as cancer-preventive compounds, generally because of their ability to neutrallize reactive oxygen species (ROS). ROS can cause damage to DNA, protein, and lipids, and overproduction can be toxic to the cell. A number of laboratories have reported that antioxidants can induce apoptosis in cells. Although thiol compounds such as NAC (Figure 4) are probably most closely associated with radical quenching, one of their most important functions is to act as cellular redox buffers by regulating protein thiol/disulfide composition. It is known that many transcription factors are active only when their sulfhydryl groups are in the reduced state. Two of the best studied of these are AP-1 and NF- $\kappa$ B. Reduced cysteine groups are important for



Figure 4. Chemical structure and natural source of N-acetylcysteine. 
the activity of $\mathrm{p} 53$, as well, potentiating its participation in apoptosis. All caspases, in addition to many other enzymes, including several src-related phosphokinases, contain cysteines in their active sites and require a reduced environment for optimal activity [76].

Radiographic contrast media is the third leading cause of hospital-acquired acute renal failure, accounting for approximately $11 \%$ of cases. The incidence of radio contrast nephropathy $(\mathrm{RCN})$ reported in the literature has ranged from $1 \%$ to $45 \%$. Diabetes mellitus and pre-existing chronic kidney disease (CKD) appear to be the most important predictors of RCN. RCN is associated with both short- and long-term morbidity and mortality. Estimates of in-hospital mortality rates are as high as $34 \%$ in patients who develop acute renal failure compared with $7 \%$ in those who do not [77].

NAC has been used in trials to equate the prevention of RCN in patients with preexisting chronic kidney disease (CKD). NAC reduces the risk for $\mathrm{RCN}$ in patients with CKD [77].

To model VEGF-dependent tumor angiogenesis in vivo and test the essential components of NAC-dependent anti-angiogenic activity, Agarwal, et al. (2004) used a VEGF-dependent angiogenesis assay in the differentiated chicken chorioallantoic membrane (CAM) [78]. When topical angiostatin was added, the overall vascular order was disrupted and microvessels appeared to abruptly terminate as evident in the gross trans-illumination images. VEGF-expressing CAMs had a high-vascular density. Treatments with either NAC or angiostatin significantly reduced the total vessel number to nearly half the level of the VEGF controls [78]. These results suggested that NAC may show potential as an anti-tumorigenic agent with efficacy in preventing initial tumor take and metastasis along with a repression of VEGF expression as shown in an experimental Kaposi's sarcoma model. NAC treatment did not repress the level of VEGF, which was still significant in hypoxic tumor microenvironments. The efficacy of NAC on the growth and viability of human breast carcinoma xenografts indicated that it is the tumor center that was predominantly affected by systemic NAC treatment, particularly with a dramatic loss of intratumoral vascular maintenance [78, 79]. Therefore, NAC antiangiogenic efficacy was shown in the "heart" of established tumors. An interesting result of the vascular depletion in the center of the tumor was that metastasis to draining lymph nodes was also affected. In addition, it has been shown that NAC has direct effects on tumor cell metastasis [80].

Antioxidants such as NAC have been known to be cytoprotective after exposure to cellular damaging agents such as reactive oxygen species. NAC is a precursor to the cellular antioxidant glutathione (GSH), a scavenger for cell and DNA-damaging oxygen species such as hy- drogen peroxide, superoxide, and lipid peroxides. In numerous studies NAC has been shown to provide significant protection for stress-related cell and genomic damage [79]. In addition, NAC has been found to be safe and efficacious in the clinic for treating acute respiratory distress and inflammation, as well as being a useful antidote for acute drug intoxication, e.g., Tylenol [81].

Sepsis remains the principal cause of mortality in patients on the intensive care unit despite improvements in supportive and antimicrobial therapies. The host response to infection or trauma is mediated by cytokines, arachidonic acid metabolites, reactive oxygen species, nitric oxide, and adhesion molecules. Although these mediators are essential for the resolution of infection and injury, prolonged production may result in host tissue and organ damage. Gene expression of these mediators is controlled in part at the transcription level via nuclear factor NF- $\kappa \mathrm{B}$. NF- $\kappa \mathrm{B}$ is present in the cytoplasm, retained in an inactive form through interaction with its inhibitory subunit, I- $\kappa \mathrm{B}$. Activation in response to lipopolysaccharide (LPS), cytokines, and other mediators occurs through a common pathway involving oxidative stress, resulting in phosphorylation of the $\mathrm{I}-\mathrm{kB}$, allowing exposure of a nuclear recognition site and migration of the active NF- $\kappa \mathrm{B}$ into the nucleus where it binds to target DNA. NF- $\alpha$ B has been shown to be involved in the upregulation of many cytokines and chemokines, including interleukin-6 (IL-6) and IL-8, and adhesion molecules, including intercellular adhesion molecule (ICAM)-1 [82]. It has been shown in numerous studies, including Uwe (2008) that NF- $\kappa \mathrm{B}$ is activated in critically ill patients, particularly in those patients who do not survive [83]. Inhibition of NF- $\kappa \mathrm{B}$ release is likely to attenuate cytokine and adhesion molecule production and therefore, may be beneficial. NF- $\kappa$ B activation and cytokine and adhesion molecule gene expression are decreased by NAC in vitro, and in various animal models of sepsis, NAC reduces adherence and chemotaxis, blunts cytokine responses, and improves survival. In critically ill patients, administration of NAC attenuates IL-8 release, increasing respiratory burst, decreases markers of free radical damage, improves oxygenation ratios, increases cardiac index and increases gastric intramucos al $\mathrm{pH}$ [82].

Paterson, et al. (2003) showed that administration of NAC in patients with severe sepsis is associated with attenuation of NF- $\kappa \mathrm{B}$ activation in mononuclear leukocytes and decreased circulating concentrations of IL-8 [82]. No effect on IL-6 of sICAM-1 was observed. An oxidative step in the activation cascade of NF- $k \mathrm{~B}$ is generally accepted, and several antioxidants have been shown to inhibit NF- $\kappa \mathrm{B}$ activation in both in vitro and in animal models [82]. Thiol antioxidants, typified by NAC, are known to induce p53-dependent apoptosis in transformed mouse embryo fibroblasts but not in normal 
mouse embryo fibroblasts while suggested that NAC may show potential as an anti-tumorigenic agent with efficacy in preventing initial tumor take and metastasis along with a repression of VEGF expression in an experimental Kaposi's sarcoma model [76].

The effect of in vivo and in vitro NAC treatment on destructive activity of macrophages against a patient's opportunistic infection with Candida received NAC (600 $\mathrm{mg}$ ) or placebo orally 3 times a day for 15 days. NAC treatment significantly enhanced antifungal activity of peripheral blood monocytes (PBM) from these patients. Long-term NAC treatment could augment resistance against microbial infections, which are often life-threatening in these patients [84].

In conclusion, NAC taken daily for a long-term period has very low toxicity and results in the improvement of biological markers which are predictive for patient outcome. Furthermore, NAC has shown its potential role in the functional restoration of the immune system in advanced cancer patients [85-89].

\section{ARABINOXYLAN}

- Increases butyrate production

- Increases natural killer cell activity

- Immune modulator

- No current phase trials

The role of dietary fiber in the prevention of colon cancer rate is still not completely understood despite numerous investigations that stemmed from Burkitt's pioneering studies in 1971 concerning the importance of dietary fibers in preventing colon cancer in humans [90]. Epidemiological studies suggest an inverse relationship between the intake of dietary fiber, particularly fiber from cereal grains, and colon cancer risk. Animal model assays have demonstrated that the protective effects of dietary fiber on colon cancer development depend on the nature and source of the fiber. Wheat bran (WB) appears to inhibit colon tumor genesis more consistently than do oat bran or corn bran. Reddy, et al. (2000) demonstrated for the first time that the lipid fraction of wheat bran has strong colon tumor inhibitor properties. Wheat bran has been shown to be the best diluter of colonic contents [91].

The Wheat Bran Fiber (WBF) trial is a Phase III clinical trial designed to assess the effect of a WBF intervenetion for 3 years on the recurrence of adenomatous polyps. It is estimated that without preventive actions, about $6 \%$ of Americans will develop colorectal cancer sometime over their lifetime. The majority of colorectal cancers arise from the premalignant lesion, the adenomatous polyp, and removal of these lesions has been shown to substantially reduce the subsequent risk for colorectal cancer. An abundant amount of research has been devoted to the study of diet in the etiology of this malign- nancy [92]. Wheat bran appears to protect against colon cancer but the mechanism(s) is not known. Possible mechanisms for wheat bran's antineoplastic effects are butyrate's enhancement of apoptosis and control of proliferation soon after carcinogen induced DNA damage to colon tissue. Apoptosis recently was reported to be a better predictor of tumor outcome than proliferation in induced carcinogenesis models. The elimination of damaged cells during tumor initiation would limit the number of aberrant crypts and tumors later in life. Control of the zone of proliferation to the lower $2 / 3$ of the crypt would decrease the number of cells lining the crypt, normalize the luminal surface and thus, limit the number of aberrant crypt foci (ACF) [93]. Poorly fermented fibers, such as wheat bran, cellulose and lignin, are protective against colon cancer. Wheat bran has shown increment in cell proliferation differences in the location of greatest butyrate concentration and alterations in luminal $\mathrm{pH}$ as a possibility for an explanation of differences between positive and negative effects between fermented and poorly fermented fibers [90,94-99]. The protective value of a fiber has often been linked to the production of butyrate and especially the concentration of butyrate in the distal colon [98]. Butyrate has long been the focal point of studies of colon physiology and pathophysiology, primarily because of its importance as the preferred source of metabolic fuel for the colonocyte [90]. A group of rats consuming diets containing oat bran at a concentration of $6 \mathrm{~g} / 100 \mathrm{~g}$ diet had greater body weights, produced larger concentrations of short-chain fatty acids, including butyrate, in both the proximal and distal colon, had more acidic luminal $\mathrm{pH}$ values, but also had a significantly larger number of animals develop colon tumors than their wheat bran counterparts [90]. There is further evidence that dietary supplements of wheat bran may protect against colon cancer. The effects of supplementing the diet of female wistar rats with $10 \%$ wheat bran on the disposition and metabolism of the dietary carcinogen 2-amino-3-methylimidazo [4,5-f] quinoline (IQ) was studied. One of the most marked effects of wheat bran was apparently to significantly retard the metabolism of IQ in the plasma [100].

In summary, supplementing the diet of both animals and humans with various dietary fiber sources is known to have a potential ability to protect against the development of cancer. Numerous animal studies indicate that supplementing the diet with wheat bran protects against colon cancer [100].

In order to find the active ingredients of bran fiber, MGN-3/Biobran, modified arabinoxylan rice bran, has been shown to be a potent biological response modifier. Results have revealed that MGN-3, in a dose dependent manner $(1,10,100 \mu \mathrm{g} / \mathrm{ml})$, significantly induced high levels of production of cytokines: TNF-alpha; and IL-6. 
In addition, MGN-3 significantly increased nitric oxide (NO) production. This data demonstrates that MGN-3 is a potent inducer of phagocytic function by macrophage, and suggests that MGN-3 is a useful agent for fighting microbial infection [101].

Arabinoxylan (Figure 5), which is a complex polysaccharide in the cereal cell wall, has been investigated as a biological response modifier. The leading manufacturer of this type of hemicellulose food supplement is Daiwa Pharmaceutical in Japan, which has a unique and patented process in which rice bran is broken down (partially hydrolyzed) using Shitake mushroom enzymes (lentius edodes mycelia extract) to make a unique and natural blend of hemicelluloses, the principal ingredient of which is the arabinoxylan compound or $\beta-1,4$ xylophyronase hemicellulose [102]. The research was performed to release and activate arabinoxylan from rice bran by using a combined process of extrusion and commercial hemicellulase. The results showed that extrusion and subsequent enzyme treatment was an industrially applicable tool for effective release of arabinoxylan with high yield and purity [103]. The non-cellulostic polysaccharides present in cereals $(2 \%-8 \% \mathrm{w} / \mathrm{w})$ are mostly arabinoxylans, $(1 \rightarrow 3),(1 \rightarrow 4)-\beta$-D-glucans, pectins, and arabinogalactins. Of these, the arabinoxylans are known to absorb large amounts of water. Nutritionally, they are classified under "unavailable carbohydrates" (dietary fiber) and are known to have beneficial effects in alleviating disease symptoms such as diabetes, atherosclerosis, and colon cancer [104]. Effects of wheat bran-derived arabinoxylans and fermentation products may act on chemoprevention [105].

In summary, the positive immunological effect of biobran may be due to the arabinoxylan release and lentinen derived from the shitake mushrooms (lentinen review).

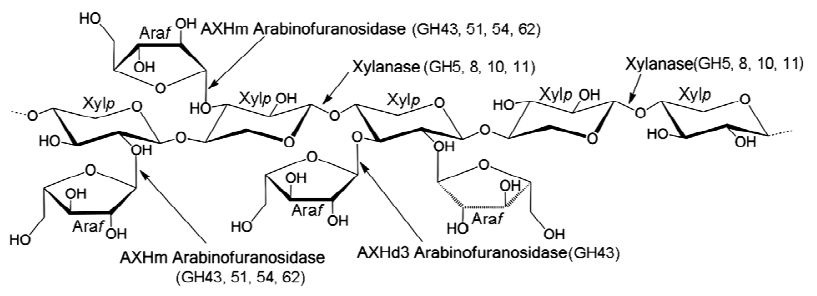

(a)

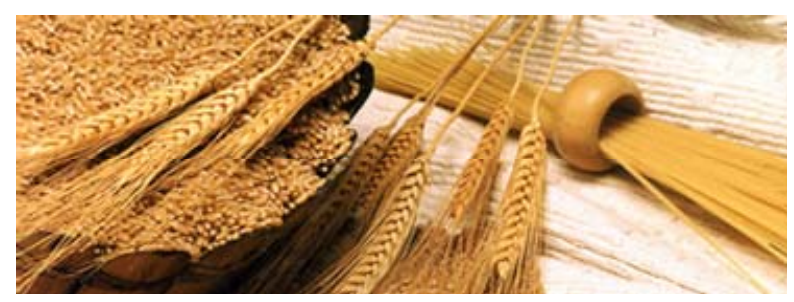

(b)

Figure 5. (a) Chemical structure of arabinoxylan (wheat); (b) Natural source of arabinoxylan (wheat).

\section{LENTINAN}

- Stimulates natural killer cells

- Immune enhancer

Numerous bioactive polysaccharides or polysaccharide-protein complexes from medicinal mushrooms are described that appear to enhance innate and cell mediated immune responses, and exhibit antitumor activities in animals and humans [106]. Of significant relevance and importance is the ability of particular mushroomderived compounds to modulate the human immune response and to inhibit certain tumor growths. Those compounds which appear to stimulate the human immune response are being sought for the treatment of cancer [107].

One of the most significant factors of many of the derived bioactive polymers from medicinal mushrooms is their role as immune modulators. The body's defense against viral attack and against spontaneously arising malignant tumor cells comprises a dynamic orchestrated interplay of innate and acquired immune responses. Innate immunity, where macrophages, neutrophils, natural killer cells (NKC) and dendritic cells are gatekeepers, is regulated by chemical-messengers or cytokines and by activation of inflammatory and acute phase responses [108]. Therefore, a fully functional immune response is critical to the recognition and elimination of tumor cells. The identification of mushroom derived compound(s) that are capable of stimulating components of innate or acquired immunities may be of potential benefit for cancer treatment.

Tumors may develop when transformed cells escape immunological host defense mechanisms [109]. Indeed, spontaneous tumors in immunosuppressed individuals indicate that the immune system can provide a significant mechanism for host resistance against cancer and infectious diseases [110,111].

Lentinan (Figure 6) is derived from a water extract of Lentinus edodes mycelium before the mushroom fruiting bodies develop and is protein-free being completely devoid of any nitrogen, phosphorous, sulphur [112].

Lentinan has proved successful in prolonging the overall survival of cancer patients, especially those with gastric and colorectal carcinoma [113]. In a randomized controlled study of patients treated with tegafur or a combination of Lentinan and tegafur overall survival was significantly prolonged in the Lentinan plus tegafur group. Of 145 patients, 68 received tegafur alone, and 77 received Lentinan plus tegafur. The respective 50\% survival times for the two groups were 92 days (tegafur alone) and 173 days (Lentinan plus tegafur). The addition of lentinan to standard chemotherapy offers a significant advantage over chemotherapy alone in terms of survival for patients with advanced gastric cancer [114]. 




Figure 6. Chemical structure and natural source of lentinan.

Among the 48 advanced colorectal cancer patients assessed for Quality of Life (QOL), the patients with low QOL scores before super fine Lentinen treatment $(\mathrm{n}=23)$ reported a significant improvement in their QOL scores after 12 weeks of SDL administration. The rates of Lentinen-binding peripheral blood lymphocytes in the QOLimproved group were significantly higher than those in the QOL-not-improved group $(\mathrm{p}<0.05)$ [115].

Lentinan does not attack cancer cells directly, but produces its antitumor effect by activating different immune responses in the host. Lentinan has displayed various kinds of immune activities in both animals and in humans. An insight into receptor-binding in immune cells by â-glucans from fungi was provided [116]. It was shown that â-glucans from yeast bind to iC3b-receptors (CR3, CD11b/CD18) of phagocytic cells and NKC, stimulating phagocytosis and cytotoxic degranulation, respectively. Lentinan can activate NKC in vitro in the same concentrations that are achieved in the blood plasma of patients treated clinically with Lentinan. Increased NKC activity is involved in tumor suppression and while these cells do not stimulate certain T-killer cell activity, or do so only under certain conditions, as strong T-helper cell stimulants both in vitro and in vivo. Lentinan can inhibit prostaglandin synthesis, which can slow T-cell differentiation in animals and humans, as well as inhibiting suppressor T-cell activity in vivo. Lentinan's immune-activating ability may be linked with its modulation of hormonal factors, which are known to play a role in tumor growth. The anti-tumor activity of Lentinan is strongly reduced by administration of thyroxine or hydrocortisone. Lentinan can also restore a tumor-specific antigen-directed delayed-type hypersensitivity (DTH) response. Interestingly, accumulating evidence suggests that Lentinan-stimulation of dendritic cells, which in- clude antigen-presenting cells that are found in lymph nodes, spleen and thymus; follicular and interdigitating dendritic cells; skin and other tissue Langerhans cells. Lentinan has an important impact on immunomodulation and anti-tumor activity. Moreover, dendritic cell tumorinfiltration in association with killer cytotoxic $\mathrm{T}$ cell stimulation and activation have been shown to have a governing role in tumor attack and elimination [117].

In Twenty-seven patients with unresectable or recurrent gastric cancer, there was a significant correlation between the QOL scores at 12 weeks (of superfine dispersed lentinan treatment and survival times [118]. Kataoka, et al. (2009) had earlier shown significant QOL scores over 12 weeks with gastric cancer patients. Both studies concluded that the combination of Lentinan from the beginning of the chemotherapy may be an important factor for the improvement of patient QOL $[118,119]$.

In summary, lentinan has been shown above in a significant number of studies to exert its anti-tumor activity by stimulating natural killer cells (NKC) and acquired immunity in their fight against virally infected cells and cancer cells. The addition of lentinan to the chemotherapy regimen improves the general condition, symptoms and signs, and quality of life of patients with EC. In particular, the patient's immune function may be enhanced by the combined treatment. The generalized application of lentinan has been recommended for clinical applications [120].

\section{ACETYL-L-CARNITINE}

- Antioxidant

- Neuroprotector and induces neurotrophy

- Increases mitochondrial metabolism

- Stabilizes intracellular membrane

- Improves fatigue symptoms

- Phase I-1

Peripheral neurotoxicity is a major complication associated with the use of chemotherapeutic agents such as platinum compounds, taxanes and vinca alkaloids [121-124]. The neurotoxicity of chemotherapy depends not only on the anticancer agent(s) used, the cumulative dose and the delivery method, but also on the capacity of the nerve to cope with the nerve-damaging process. The sensory and motor symptoms and signs of neurotoxicity are disabling, and have a significant impact on the QOL of cancer patients. Moreover, the risk of cumulative toxicity may limit the use of highly effective chemotherapeutic agents. Therefore, prophylaxis and treatment of peripheral neurotoxicity secondary to chemotherapy are major clinical issues. Acetyl-L-carnitine (ALC) plays an essential role in intermediary metabolism (Figure 7). Some of the properties exhibited by ALC include neuroprotective and neurotrophic actions, antioxidant activity, 


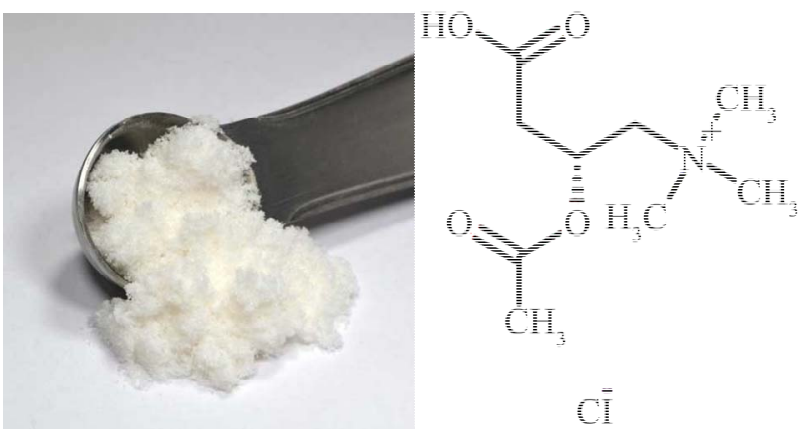

Figure 7. Chemical structure of Acetyl-L-carnitine.

positive actions on mitochondrial metabolism, and stabilization of intracellular membranes. ALC has demonstrated efficacy and high tolerability in the treatment of neuropathies of various aetiologies, including chemotherapy-induced peripheral neuropathy (CIPN). In several experimental settings, the prophylactic administration of ALC prevented the occurrence of peripheral neurotoxicity commonly induced by chemotherapeutic agents. In animal models of CIPN, ALC administration promoted the recovery of nerve conduction velocity, restored the mechanical nociceptive threshold, and induced analgesia by up-regulating the expression of type- 2 metabotropic glutamate receptors in dorsal root ganglia. These results, plus the favorable safety profile of ALC in neuropathies of other aetiologies, have led to the effects of ALC on CIPN being investigated in cancer patients. Preliminary results have confirmed the reasonably good tolerability profile and the efficacy of ALC on CIPN. ALC has several mechanisms, which include the regeneration of injured nerve fibers, reducing oxidative stress, supporting DNA synthesis in mitochondria and enhanceing nerve growth factor concentrations in neurons [121]. Current studies support the use of ALC in cancer patients with persisting neurotoxicity induced by paclitaxel or cisplatin treatment [122].

Acetyl-L-carnitine (ALC) enhances neurotrophic support of sensory neurons, potentially causing symptom relief and nerve regeneration, and in addition has numerous other effects on metabolic function that might be of benefit in such patients. ALC has been given to HIV patients with symptomatic ATN in a number of clinical studies $[123,125]$.

Diabetic polyneuropathy (DPN) is the most common late complication of diabetes mellitus. Clinical trials utilising ALC have shown beneficial effects on nerve conduction slowing, neuropathic pain, axonal degenerative changes and nerve fibre regeneration, despite relatively late initiation in the natural history of DPN. Owing to the good safety profile of ALC, early initiation of ALC therapy would be justified, with potentially greater benefits. [124].

Soy isoflavones and L-carnitine, stimulate carnitine palmitoyl transferase $1 \mathrm{~A}$ and a cofactor for beta-oxidation of fatty acids, respectively, thus enhancing fatty acid oxidation. These results suggest that these compounds may be effective in controlling obesity [126].

Fatigue is the most commonly reported symptom in patients with cancer, with a prevalence of over $60 \%$ reported in the majority of studies. Clinical trials that assessed pharmacologic agents for the treatment of cancer related fatigue include ALC [127].

Nucleoside reverse transcriptase inhibitors disrupt neuronal mitochondrial DNA synthesis, resulting in antiretroviral toxic neuropathy (ATN). ALC enhances neurotrophic support of sensory neurones, potentially providing symptom relief and nerve regeneration. ALC, administered twice a day intramuscularly to HIV-1-infected patients with symptomatic ATN, significantly reduced weekly mean pain ratings compared with a placebo. Oral ALC even improved symptoms. Intramuscular and oral ALC was generally safe and well tolerated $[123,125]$. Carnitine deficiency is among the many metabolic disturbances that may contribute to fatigue in patients with cancer [128]. Administration of ALC may hold promise as a treatment for this common symptom as shown in Phase I/II trials to assess the safety and tolerability of exogenous ALC and clarify the safe dose range associated with symptom effects for future controlled trials. Of the 38 patients screened for carnitine levels, 29 were deficient $(76 \%)$. The highest dose used in these studies was $3000 \mathrm{mg} /$ day. No patient experienced significant side effects and no toxicities were noted. These findings suggest that ALC may be safely administered at doses up to $3000 \mathrm{mg}$ where positive effects may be more likely to occur at [129]. Treatments for cancer-related fatigue with an aim to develop directions for future research in large, randomized clinical trials [130].

Supplementation with ALC does not impair the ability of epirubicin to kill breast cancer cells. These results suggest that supplementation with ALC in patients undergoing epirubicin treatment could be safely used to reduce associated cardiotoxicities without fear that the efficacy of chemotherapy is jeopardized [131].

Recent publications have linked oxidative stress to a variety of upper gastrointestinal insults. ALC prevents the oxidative stress response and hold great promise for antioxidant compounds that are safe, efficacious, and inexpensive [132].

Lack of sufficient levels of ALC is among the postulated causes of fatigue, a highly prevalent symptom in the multiple sclerosis (MS) population, which has a serious impact on patients' quality of life. Deficiency of carnitine may play a role by reducing energy production through fatty acid oxidation and numerous MS therapies can induce fatigue syndrome. For $63 \%$ of patients treated with immunosuppressive or immunomodulatory thera- 
pies, oral ALC decreased fatigue intensity, especially in patients treated with cyclophosphamide and interferon beta [133].

\section{ALPHA-LIPOIC ACID}

- Antioxidant

- Increases glutathione peroxidase

- Apoptosis

- Inhibits proliferation of cancer cells

- Phase I-1

- Phase II-2

The antioxidant alpha-lipoic acid (APA) is a naturally occurring compound (Figure 8) that has been shown to posses promising anti-cancer activity because of its ability to preferentially induce apoptosis and inhibit proliferation of cancer cells relative to normal cells.

Mantovani, et al. (2002) used alpha-lipoic acid (ALA) at a dosage of $300 \mathrm{mg} /$ day and $\mathrm{N}$-acetylcysteine at 1800 $\mathrm{mg} /$ day [89]. Their data showed long term combined maintenance therapy with rIL $2+$ medroxyprogesterone acetate (MPA) + antioxidant agents is feasible, has a very low toxicity, and results in the improvement of clinical outcome [134]. The antioxidants N-Acetylcysteine and ALA markedly reduced the effect of the hormone on tumor necrosis factor-induced caspase activation, attesting to the involvement of reactive oxygen species (ROS) in the cross-talk between the hormone and the cytokine [135]. Mantovani, et al. (2003) [136] tested the ability of different antioxidant agents, used alone or in combination, to reduce the reactive oxygen species (ROS) levels and to increase the glutathione peroxidase (GPx) activity. The study included fifty-six advanced stage cancer patients who were mainly stage III (12.5\%) and stage IV (82.1\%). Single antioxidants were effective in reducing the ROS levels.

The results of ALA use in human cancer chemotherapy and as a chemo-preventive agent by a significant inhibition of the formation of the depurinating adducts [137] have been reviewed in light of ALA future inclusion into chemotherapeutic protocols [138,139]. The efficacy, the apparent lack of toxicity, the long clinical track records of these medications in human medicine, all points toward the need for a clinical trial. The dramatic efficacy of treatment suggests that cancer may

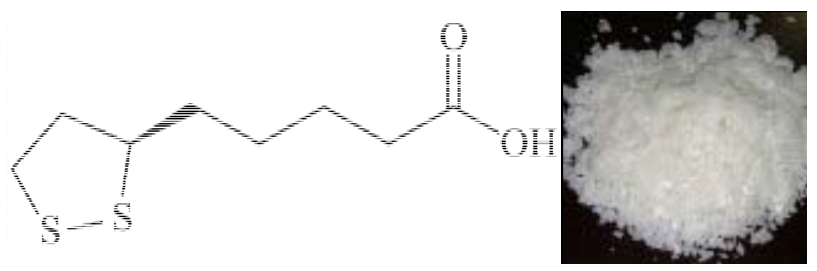

Figure 8. Chemical structure and natural source of alpha-lipoic acid. simply be a disease of dysregulated cellular metabolism [140].

Moungjaroen, et al., 2006 showed ALA induced reactive oxygen species (ROS) generation and a concomitant increase in apoptosis of human lung epithelial cancer H460 cells [141]. Apoptosis induced by ALA was found to be mediated through the mitochondrial death pathway, which requires caspase-9 activation. A phase II study with ALA showed efficacy and safety in patients with cancer-related anorexia/cachexia and oxidative stress [85]. An open early-phase II study was designed with 39 patients given $300 \mathrm{mg} /$ day ALA and treatment duration for 4 months. There was an important decrease of proinflammatory cytokines interleukin-6 (IL-6) and tumor necrosis factor-alpha, and a negative relationship worthy of note was only found between LBM and IL-6 changes. As for quality of life evaluation, there was a marked improvement. At the end of the study, 22 of the 39 patients were "responders" or "high responders." They concluded efficacy and safety of the treatment have been shown by the study; therefore, a randomized phase III study is warranted.

Menopause is often accompanied by hot flashes and degenerative processes such as arteriosclerosis and atrophic changes of the skin that suggest an acceleration of aging triggered by estrogen lack. Therefore, hormone replacement therapy (HRT) has been considered the most suitable treatment for the above symptoms and processes. However, because of the possible serious side effects of HRT (especially the increased risk of thrombo-embolic accidents and breast cancer) there is a growing demand for alternative treatments of the symptoms and pathological processes associated with menopause. Oxygen stress contributes to menopause and some of its physiopathological effects may be prevented and/or treated by improving the antioxidant defense. Antioxidants, including ALA, have favorable effects on the health and QOL of women, especially those who cannot be treated with HR or who suffer from high levels of oxygen stress [142, 143].

In summary, the efficacy, the apparent lack of toxicity, the long clinical track records of using ALA in human medicine, all point toward the need for clinical trials. The dramatic efficacy of ALA treatment suggests that cancer may result in part as a function of dysregulated cellular metabolism [140].

\section{COENZYME Q10}

- Antioxidant

- Immune stimulator

- Increases energy

- Increases oxygen

- Increases circulation

- Anti-aging properties 
- Phase I trials-4

- Phase II trials-1

Coenzyme Q10 (CoQ10, ubiquinone) is a compound whose actions resemble those of vitamin E (Figure 9). It is stored in the fatty tissues of the body, reducing the need to ingest large quantities. Since CoQ10 is fat soluble, it is best absorbed when taken with oily or everywhere in the body. It stimulates the immune system, aids circulation, increases tissue oxygenation, and has antiaging effects. It has the ability to counter histamine, and therefore, is beneficial for people with allergies, asthma, or respiratory disease. It has been used to treat schizophrenia and Alzheimer's disease and is also beneficial in fighting obesity, multiple sclerosis, and diabetes. More than 12 million people in Japan are reportedly taking it at the direction of their physicians for treatment of heart disease. The amount of CoQ10 present in the body declines with age, so it should be supplemented in the diet, especially for those over the age of fifty.

Antioxidants are emerging as prophylactic and therapeutic agents. These are the agents, which scavenge free radicals otherwise reactive oxygen species and prevent the damage caused by them. Free radicals have been associated with pathogenesis of various disorders like cancer, diabetes, cardiovascular diseases, autoimmune diseases, neurodegenerative disorders and are implicated in aging. Several antioxidants like SOD, CAT, epigallocatechin-3-O-gallate, lycopene, ellagic acid, coenzyme $\mathrm{Q} 10$, indole- 3-carbinol, genistein, quercetin, vitamin $\mathrm{C}$ and vitamin $\mathrm{E}$ have been found to be pharmacologically active as prophylactic and therapeutic agents for above mentioned diseases. Results from several studies are positive [144]. Matkovics, 2006 recommends a combination in which physiological amounts of vitamins C, D, K and B-complex, N-Acetylcysteine, vitamin $\mathrm{E}$ of natural origin might be complemented by allopurinol, co-enzyme Q-10 and alpha-lipoic acid [145].

CoQ10's role as an antioxidant may be more powerful than Vitamin E especially in preventing oxidation of LDL Cholesterol. Antioxidants help the body deal with unstable chemicals called free radicals. Free radicals are produced by the body when food is converted into energy and will build up in the body over time. They increase the potential for damage to the body cells (a process called oxidative stress), which is associated with the

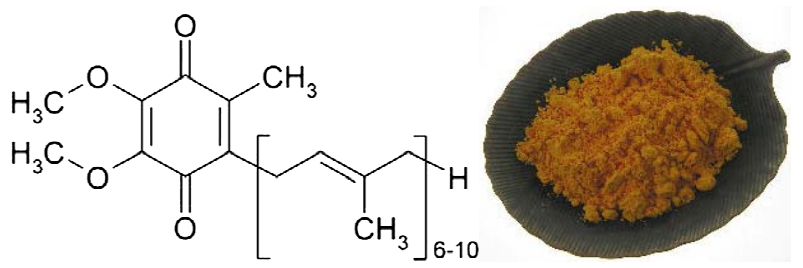

Figure 9. Chemical structure and natural source of coenzyme Q10. aging process and a general decline in the central nervous system and the immune system. They are also thought to contribute to the development of various health conditions such as cancer, heart disease, and inflamemation conditions for example arthritis. Furthermore, antioxidants can help to prevent the conversion of nitrates found in tobacco smoke, bacon, and some vegetables into cancer-causing substances.

CoQ10 is an essential cofactor in the electron transport chain, serves as a potent antioxidant in mitochondria and lipid membranes, and is often used as a dietary supplement for a number of diseases including cardiovascular diseases. The identified CoQ10-inducible genes and pathways play an important role in inflammatory response [146]. Due to the notable absence of clinically significant side effects and likely therapeutic benefit, CoQ10 can be considered a safe adjunct to standard therapies in cardiovascular disease [147]. Preoperative oral CoQ10 therapy in patients undergoing cardiac surgery increases myocardial and cardiac mitochondrial CoQ10 levels, improves mitochondrial efficiency, and increases myocardial tolerance to in vitro hypoxia-reoxygenation stress [148].

Bailey (2007) showed that acute exercise increased free radical formation in human skeletal muscle [149]. These findings provide the first direct evidence for intramuscular free radical accumulation and lipid peroxidation following acute exercise in humans.

Statins inhibit the production of CoQ10, which is required for mitochondrial electron transport [150]. Fifty consecutive new cardiology clinic patients who were on statin drug therapy (for an average of 28 months) on their initial visit were evaluated for possible adverse statin effects (myalgia, fatigue, dyspnea, memory loss, and peripheral neuropathy). All patients discontinued statin therapy due to side effects and began supplemental CoQ10 at an average of $240 \mathrm{mg} /$ day upon initial visit. Patients have been followed for an average of 22 months with $84 \%$ of the patients followed now for more than 12 months. The prevalence of patient symptoms on initial visit and on most recent follow-up demonstrated a decrease in fatigue from $84 \%$ to $16 \%$, myalgia from $64 \%$ to $6 \%$, dyspnea from $58 \%$ to $12 \%$, memory loss from $8 \%$ to $4 \%$ and peripheral neuropathy from $10 \%$ to $2 \%$. They concluded that statin-related side effects, including statin cardiomyopathy, are far more common than previously published and are reversible with the combination of statin discontinuation and supplemental CoQ10, and they saw no adverse consequences from statin discontinuation [151]. Statin drugs (3-hydroxy-3-methylglutaryl coenzyme A reductase inhibitors) reduce the level of cholesterol by inhibiting the synthesis of mevalonate, an intermediary in the cholesterol biosynthetic pathway. Use of statin drugs has been associated with a variety of skeletal 
muscle-related complaints. CoQ10, a component of the mitochondrial respiratory chain, is also synthesized from mevalonate, and decreased muscle CoQ10 concentration may have a role in the pathogenesis of statin drug-related myopathy [152]. Statins decrease LDL cholesterol and the risk of atherosclerotic cardiovascular disease (CVD). They also decrease CoQ10, an effect that may negate some of the statin benefit on CVD [153].

An essential role of coenzyme Q10 is as an electron carrier in the mitochondrial respiratory chain. Moreover, CoQ10 is one of the most important lipophilic antioxidants, preventing the generation of free radicals as well as oxidative modifications of proteins, lipids, and DNA. It can also regenerate the other powerful lipophilic antioxidant, alpha-tocopherol. Decreased levels of CoQ10 in humans are observed in many pathologies (e.g. cardiac disorders, neurodegenerative diseases, AIDS, cancer) associated with intensive generation of free radicals and their action on cells and tissues. In these cases, treatment involves pharmaceutical supplementation or increased consumption of CoQ10 with meals [154].

Lowering of low-density lipoprotein cholesterol is well achieved by coenzyme Q10. Statins inhibit the production of CoQ10 play an important role in statin-induced hepatopathy. CoQ10 supplementation protects cells from this complication and should be taken if statins are prescribed [155].

Neuronal cell death induced by oxidative stress is correlated with numerous neurodegenerative diseases, including Alzheimer's disease (AD), Parkinson's disease (PD), and stroke. Paraquat, a nonselective herbicide, was once widely used in North America and is still routinely used in Taiwan. Results indicate that water-soluble CoQ10 can prevent oxidative stress and neuronal damage induced by paraquat and therefore, can be used for the prevention and therapy of neurodegenerative diseases caused by environmental toxins [156]. Furthermore, the manual workers of the gas-and-oil extraction industry (Russian Siberian extraction plants) are exposed to hostile environmental and occupational conditions, resulting in elevated mortality and disability, due to chronic neurological and cardiovascular diseases. The short term administration of a nutraceutical formulation based on CoQ10, vitamin E, selenium, methionine and phospholipids led to significant improvement of cardiovascular parameters and psycho-emotional status, consistent with the normalization of LDCL and peroxynitrite production by WBC, with a good compliance to treatment confirmed by the increased blood levels of ubiquinol [157]. Finally, UV-C radiation is able to impair cellular functions by directly damaging DNA and by inducing an increased formation of reactive oxygen species that leads to a condition of oxidative stress. Intracellular levels of ROS, mitochondrial depolarization and cell viability was meas- ured by flow cytometry [158]. Enhancing CoQ10 synthesis and suppressing the induction of NF- $\kappa \mathrm{B}$, may provide neuroprotection [159].

Brain aging and neurodegenerative disorders involve impaired energy metabolism and oxidative damage, but the involvement of the Plasma Membrane Redox System (PMRS) in these processes is unknown. Caloric restriction protects the brain against aging and disease by increasing the activities of PMRS. These findings suggest important roles for the PMRS in protecting brain cells against age-related increases in oxidative and metabolic stress [160]. Enhancing CoQ10 synthesis and suppressing the induction of $\mathrm{NF}-\kappa \mathrm{B}$, may provide neuroprotection [159].

The feasibility of using a coupled in vitro digestionCaco-2 cell uptake as a model for examining the digestive stability and absorption of CoQ10 from a variety of commercially available CoQ10 products was examined. The CoQ10 uptake by the cells was correlated with the extent of micellarization of CoQ10 during simulated digestion. Most of CoQ10 taken up by the cells was converted to ubiquinol either during or following uptake [161].

Coenzyme Q10 is used by the body as an endogenous antioxidant. This property combined with its essential function in mitochondrial energy production suggests that it may have therapeutic potential in cancer treatment. As part of the body's antioxidant defense against free radical production, CoQ10 concentrations may change during anti-cancer chemotherapy. CoQ10 was measured in the plasma of 27 children with acute lymphoblastic leukemia (ALL) at the time of diagnosis, during induction (protocol ALL-BFM 2000), and post induction treatment. The starting values were compared to the CoQ10 concentrations in 92 healthy children. The total CoQ10 concentration and its redox status were measured by HPLC using electrochemical detection and internal standardization. While the CoQ10 concentration in the plasma of children with ALL was within a normal range at the time of diagnosis $(0.99+/-0.41 \mathrm{pmol} / \mathrm{ml})$, a drastic increase was observed during induction treatment $(2.19$ $+/-1.01 \mathrm{pmol} / \mathrm{ml}$ on day 33 ). This increase was accompanied by shift in the redox status in favor of the reduced form of CoQ10. The increase in CoQ10 concentration during induction treatment may be attributed to the activation of a natural antioxidative defense mechanism [162].

Anthracyclines are among the most effective chemotherapeutic agents in the treatment of numerous malignancies. Unfortunately, their use is limited by a dosedependent cardiotoxicity [163]. Preclinical and clinical studies suggest that anthracycline-induced cardiotoxicity can be prevented by administering CoQ10 during cancer chemotherapy that includes drugs such as doxorubicin 
and daunorubicin. Studies further suggest that CoQ10 does not interfere with the antineoplastic action of anthracyclines and might even enhance their anticancer effects. CoQ10, an essential component of the electron transport system and a potent intracellular antioxidant, appears to prevent damage to the mitochondria of the heart, thus preventing the development of anthracycline-induced cardiomyopathy [164].

Anthracycline-induced cardiotoxicity after treatment for childhood cancer is a serious problem [163]. Dexrazoxane prevents or reduces cardiac injury without compromising the antileukemic efficacy of doxorubicin, and CoQ10 showed a strong protective effect on cardiac function during anthracycline therapy [165].

The prognostic significance of supplementing CoQ10, riboflavin and niacin (CoRN) along with tamoxifen to breast cancer patients was evaluated by measuring the serum cytokine levels of interleukin (IL)-1beta, IL-6, IL-8, tumor necrosis factor alpha (TNF-alpha) and vascular endothelial growth factor. In this study, 84 breast cancer patients were randomized to receive a daily supplement of CoQ10 $100 \mathrm{mg}$, riboflavin $10 \mathrm{mg}$ and niacin $50 \mathrm{mg}$, one dosage per day along with tamoxifen $10 \mathrm{mg}$ twice a day. Serum cytokine levels were elevated in untreated breast cancer patients (Group II) and significantly reduced after tamoxifen therapy for more than 1 year (Group III). CoRN supplementation in breast cancer patients suggested a good prognosis and efficacy of the treatment, and might even offer protection from metastases and recurrence of cancer [166]. Hertz, Lister (2011) studied the survival of patients with end-stage cancer who received supplements of CoQ10 and a mixture of other antioxidants (e.g. vitamin C, selenium, folic acid and beta-carotene). During a period of 9 years, 41 patients who had end-stage cancer were included, in which $40 \%$ lived longer than the median predicted survival.

There is an inverse relationship between circulating CoQ10 and breast cancer riskoxifen (TAM), a non-steroidal anti-estrogen that is widely used in adjuvant therapy for all stages of breast carcinomas and in chemoprevention of high-risk group. The hepatic estrogenic effect of TAM induces hypertriglyceridemia by reduced activity of lipolytic enzymes (LPL) on triglycerides. CoQ10, riboflavin and niacin are proved to be potent antioxidant and protective agents against many diseases including cancer and cardiovascular diseases (CVD). The study figures the altered lipid and lipoprotein levels in the untreated and TAM-treated breast cancer patients. On combination therapy with Co Q10, riboflavin and niacin, it counteracts the tamoxifen-induced hyperlipidemia to normal levels [167].

The influence of menopause and hormone replacement therapy (HRT) on serum levels of CoQ10 and other lipidsoluble antioxidants in normal women has been studied.
Serum levels of CoQ10, alpha-tocopherol, gamma-tocopherol, beta-carotene and lycopene were determined in 50 premenopausal women (not using oral contraceptives), 33 healthy postmenopausal and 15 postmenopausal women on HRT. The decrease in serum concentrations of CoQ10 produced by HRT, promotes oxygen free radicalinduced membrane damage and may result in cardiovascular risk in postmenopausal women using HRT [168].

Early surgical intervention remains the most successful therapy for melanoma. Despite better outcomes observed in soft tissue and lymph node metastases, the results of pharmacological therapies are still disappointing. This study involved patients with stages I and II melanoma (American Joint Committee on Cancer criteria 2002) and surgically removed lesions. Treatment efficacy was evaluated as incidence of recurrences at 5 years.

Long-term administration of an optimized dose of recombinant interferon alpha- $2 \mathrm{~b}$ in combination with coenzyme Q10 seemed to induce significantly decreased rates of recurrence and had negligible adverse effects [169]. Abnormally low plasma levels of coenzyme CoQ10 have been found in patients with cancer of the breast, lung, or pancreas. Analysis of baseline plasma CoQ10 levels is a powerful and independent prognostic factor that can be used to estimate the risk for melanoma progression [170].

Free radicals have been implicated in the action of many chemotherapeutic drugs. Camptothecin and other chemotherapeutic drugs, such as etoposide, doxorubicin, and methotrexate, induce an increase in CoQ10 levels as part of the antioxidant defense against free radical production under these anticancer treatments in cancer cell lines. Chemotherapy treatment induced both free radical production and an increase in CoQ10 levels in all the cancer cell lines tested. Reduced CoQ10 form levels were particularly enhanced. Findings suggest that CoQ10 increase is implicated in the cellular defense under chemotherapy treatment and may contribute to cell survival [171].

Suggestions that CoQ10 might reduce the toxicity of cancer treatments have not been tested by rigorous trials. Further investigations are necessary to determine whether CoQ10 can improve the tolerability of cancer treatments [172].

In summary, CoQ10 plays a critical role in the production of energy in every cell of the body. It aids circulation, stimulates the immune system, increases tissue oxygenation, and has anti-aging effects. CoQ10 is an essential cofactor in the electron transport chain, serves as a potent antioxidant in mitochondria and lipid membranes. Statins inhibit the production of CoQ10 and thus, play an important role in statin-induced hepatopathy. CoQ10 supplementation protects cells from this complication. Finally, CoQ10 is used as a dietary supplement for a 
number of diseases including cardiovascular diseases and cancer.

\section{RESVERATROL}

- Antioxidant

- Induced apoptosis

- Inhibits cell growth

- Anticoagulant

Resveratrol (Figure 10) is a phytoalexin produced naturally by several plants when under attack by pathogens such as bacteria or fungi. Resveratrol (3,4',5-trihydroxystilbene) is found in various plants, including grapes, berries and peanuts. It is also present in wines, especially red wines.

There is mounting evidence in the treatment of a variety of human cancers that resveratrol has preventative and anti-cancer activity [173]. Resveratrol is a potential candidate due their ability to regulate multiple survival pathways without inducing toxicity [174]. The strong link between inflammation and colorectal carcinogenesis provides the rationale for using anti-inflammatory agents for chemoprevention of colorectal cancer [175].

Resveratrol is known to have potent anti-inflammatory and antioxidant effects and to inhibit platelet aggregation and the growth of a variety of cancer cells. Its potential chemopreventive and chemotherapeutic activities have been demonstrated in all three stages of carcinogenesis (initiation, promotion, and progression), in both chemically and UVB-induced skin carcinogenesis in mice, as well as in various murine models of human cancers [176]. Furthermore,

Resveratrol inhibits both $\mathrm{NF}-\kappa \mathrm{B}$ and AP-1 mediated MMP-9 expression, leading to suppression of migration and invasion of human metastatic lung and cervical cancer cells. Resveratrol has potential for clinical use in preventing invasion by human metastatic lung and cervical cancers. Resveratrol could be used to sensitize cancer cells to cell death in combination with anticancer drugs [177-179].

Breast cancer is one of the most common types of cancer in women, and is the second leading cause of cancer-related deaths in the United States. Chemoprevention using phytoestrogens, such Resveratrol and Genistein, for breast cancer may be a valid strategy [180].


Figure 10. Chemical structure and natural source of resveratrol.
Resveratrol has been shown to have positive effects on age longevity, lipid levels and a preventative quality against certain cancers and viral infections. Resveratrol induces apoptosis by up regulating the expression of Bax, Bak, PUMA, Noxa, Bim, p53, TRAIL, TRAIL-R1/DR4 and TRAIL-R2/DR5 and simultaneously down-regulating the expression of Bcl-2, Bcl-XL, Mcl-1 and survivin. Resveratrol has also been shown to reduce inflammation via inhibition of prostaglandin production, cyclooxygenase- 2 activity, and NF- $\kappa \mathrm{B}$ activity. Modulation of cell signaling pathway by resveratrol explains its diverse bioactivities related with human health. Resveratrol also potentiates the apoptotic effects of cytokines, chemotherapeutic agents and $\gamma$-radiation. The main target organs of resveratrol are liver and kidney, and it is metabolized by hydroxylation, glucuronidation, sulfation and hydrogenation. As a chemoprevention agent, resveratrol has been shown to inhibit tumor initiation, promotion, and progression. There is growing evidence that resveratrol can prevent or delay the onset of various cancers, heart diseases, ischemic and chemically induced injuries, pathological inflammation and viral infections [181].

A phase I study of oral resveratrol (single doses of 0.5 , $1,2.5$, or $5 \mathrm{~g}$ ) was conducted in 10 healthy volunteers per dose level. Consumption of resveratrol did not cause serious adverse events at the highest levels, with peak plasma levels occurring at $1.5 \mathrm{~h}$ post-dose. Cancer chemopreventive effects of resveratrol in cells in vitro require levels of at least $5 \mu \mathrm{mol} / \mathrm{L}$ [182].

Resveratrol appears to be a good candidate in chemopreventive or chemotherapeutic strategies and is believed to be a novel weapon for new therapeutic strategies [183].

It is conceivable to design Resveratrol containing emollient or patch, as well as sunscreen and skin-care products for prevention of skin cancer and other conditions, which are believed to be caused by UV radiation [184].

Resveratrol is a phytoalexin produced naturally by several plants when under attack by pathogens such as bacteria or fungi. Resveratrol is found in various plants, including grapes, berries and peanuts. It is also present in wines, especially red wines. During the last years, it has been the focus of numerous in vitro and in vivo studies investigating its biological attributes, which include mainly antioxidant [185-187] and anti-inflammatory activities, anti-platelet aggregation effect [188] and chemoprevention $[181,189]$.

In fact, recently, it has been demonstrated that the stilbene blocks the multistep process of carcinogenesis at various stages: tumor initiation, promotion and progresssion [190,191].

More recent results provide interesting insights into the effect of this compound on the life span of yeasts and 
flies, implicating the potential of resveratrol as an anti aging agent in treating age-related human diseases. Resveratrol has the potential to act as an estrogen agonist or antagonist depending on such factors as cell type, estrogen receptor isoform (ER alpha or ER beta), and the presence of endogenous estrogens [192] reports an inverse relationship between circulating CoQ10 and breast cancer risk.

In summary, the search for novel and effective cancer chemopreventive agents has led to the identification of various naturally occurring compounds one of which is resveratrol, a phytoalexin derived from the skin of grapes and other fruits. Resveratrol is known to have potent anti-inflammatory and antioxidant effects and to inhibit platelet aggregation and the growth of a variety of cancer cells. Its potential chemopreventive and chemotherapeutic activities have been demonstrated in all three stages of carcinogenesis (initiation, promotion, and progression), in both chemically and UVB-induced skin carcinogenesis in mice, as well as in various murine models of human cancers. Evidence from numerous in vitro and in vivo studies has confirmed its ability to modulate various targets and signaling pathways. This review discusses the current preclinical and mechanistic data available and assesses resveratrol's anticancer effects to support its potential as an anticancer agent in human populations.

\section{SELENIUM}

- Antioxidant

- Induces Apoptosis

- Reduces Inflammation

Selenium (Figure 11) is involved in various biological processes in nearly all tissues of animals and human, e.g. protection against oxidative stress in the cardiovascular system, and may play a role in cancer protection [193]. Selenoprotein $\mathrm{P}(\mathrm{SeP})$ is a highly glycosylated plasma protein containing up to 10 selenocysteine residues. It is secreted by hepatocytes and also by the human hepatoma cell line HepG2 [194].

The precise mechanisms of apoptosis induced by various selenium compounds are not well understood. Sodium selenite induced apoptosis is accompanied by increased Bax expression [195]. A combination of low Se



Figure 11. Chemical structure and natural source of selenium. intake and SNP in selenoprotein genes can impair that role and so lead to increased risk of pre-neoplastic lesions [196]. Bardia, et al. (2008) showed association between antioxidant use and primary cancer incidence and mortality and to evaluate these effects across specific antioxidant compounds [197]. Selenium supplementation might have anticarcinogenic effects in men and thus, requires further research. Doxorubicin and selenium cooperatively activate Fas signaling leading to apoptosis [198]. The use of antioxidants during chemotherapy has been shown to reduce or prevent the undesirable effects experienced by healthy cells. Micronutrient selenium is well known for its antioxidant properties. Selenomethionine is effective in reducing the genetic damage induced by the antitumoral agent doxorubicin [199].

Prostate cancer is the most commonly diagnosed malignancy in males. Cheung, et al. (2008) showed the natural products with the greatest potential to reduce the risk of prostate cancer, including lycopene, vitamin E, selenium, vitamin D, soy and green tea [200].

Although recent reports suggest that selenium can modulate the activity of cytotoxic drugs, the mechanism underlying this activity remains unclear. This has been investigated using a panel of human B-cell lymphoma cell lines. Taken together, these results show that the NF- $\kappa$ B pathway is one target for methylselenic acid (MSA) underlying the interaction between MSA and chemotherapy [201].

Selenium is incorporated in the proteome in the form of the genetically encoded amino acid selenocysteine, which is the characteristic component of the selenoproteins. Bartel, et al. (2007) investigated the expression of the selenoenzyme GPx-2, which is predominantly present in the tissues of the gastrointestinal tract such as the small intestine and therefore named gastrointestinal glutathione peroxidase [193]. The GPx-2 activity in the Sedeficient rat colon samples was 6.8 fold lower than in the Se-adequate rats in contrast to 1.2 fold lower levels between the corresponding samples in the small intestine. This finding might explain the different susceptibility of the colon and the small intestine to cancer and support the theory of the protective effect of selenium in the gastrointestinal tract [193].

Damage to DNA and other cellular molecules by reactive oxygen species ranks high as a major culprit in the onset and development of colorectal cancer. Expression of 14 oxidative stress-related molecules in both tumorous and non-tumorous tissues in 41 patients was examined by immunohistochemistry and Western blot analysis. These data suggest that contrasting expression pattern of the antioxidant selenoproteins plays an important role in the progression of colorectal cancer [202].

A 14.7-year follow-up for gastric cancer incidence and cause-specific mortality among 3365 randomly assigned 
subjects in a masked factorial placebo-controlled trial was conducted using selenium among other vitamins and garlic. Garlic and vitamin treatments were associated with non-statistically significant reductions in gastric cancer incidence and mortality. Vitamin treatment was associated with statistically significantly fewer deaths from gastric or esophageal cancer, a secondary endpoint $(\mathrm{HR}=0.51,95 \% \mathrm{CI}=0.30$ to $0.87 ; \mathrm{P}=0.014)$ [203].

Most known chemopreventive agents including certain selenium compounds suppress the activation of the NF$\kappa \mathrm{B}$ [204]. The meta-analysis of randomized controlled trials indicates that there is possible evidence to support the use of selenium supplements alone for cancer prevention in the low baseline serum selenium level population and in the high-risk population for cancer (Lee, et al. 2011 [205]). A number of clinical trials in recent years have provided convincing evidence of the central role of selenium, either alone or in combination with other micronutrients or antioxidants, in the prevention and treatment of multiple diseases [206].

Selenium methylselenocysteine (Se-MeSeCys) is a common selenocompound in the diet with a tested chemopreventive effect. Cuello, et al. (2007) showed that treatment of HepG2 cells with concentrations of Se$\mathrm{MeSeCys}$ in the nanomolar to micromolar range confers a significant protection against an oxidative insult [207].

The thyroid gland has an exceptionally high selenium content, even during selenium deficiency. At least 11 selenoproteins are expressed, which may be involved in the protection of the gland against the high amounts of $\mathrm{H}_{2} \mathrm{O}_{2}$ produced during thyroid hormone biosynthesis [208].

In summary, Methylselenocysteine has shown to be chemopreventative effect in a variety of diseases as a protective vitamin.

\section{ZINC}

\section{- Antioxidant}

Zinc (Figure 12) is an essential trace element for human health and is a critical component of over 300 enzymes and transcription factors involved in DNA damage response and repair [209]. The prostate is known to accumulate high levels of zinc, but levels are markedly

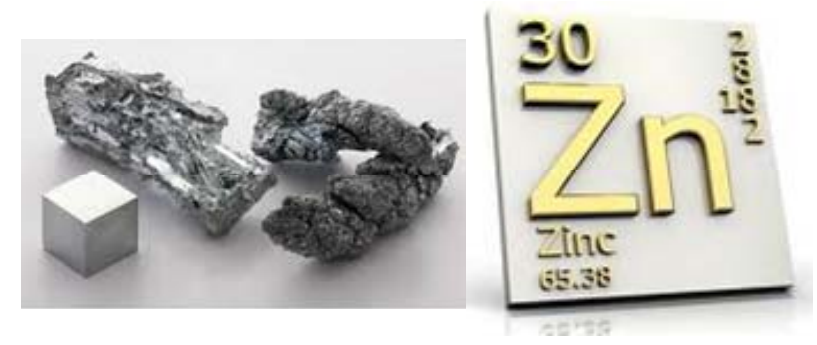

Figure 12. Chemical structure and natural source of zinc. decreased with cancer development. Zinc deficiency may compromise DNA integrity in the prostate by impairing the function of zinc-containing proteins [210].

In summary, we are not going to cure cancer with one drug, and it is very difficult to have two drugs approved together by the FDA. Our approach is to use natural, over-the-counter compounds that have showed safety and efficacy in the scientific literature with most undergoing FDA phase trials. However, these compounds are only for health and wellness and are advocated for the treatment or cure of any disease. One will gather their own information from a careful review of the literature.

There have been a number of very large studies that have advocated neutraceuticals for prevention while others have been critical. The problems with large scale "survey" studies are that they do require a questionnaire, and even the best monitored studies suffer from the decreasing interest of the participants over time affecting a true outcome. We can also question the purpose of these studies. For example, a statement such as it is recommended that wide-spread use of antioxidant vitamins in cardiovascular protection should not be instituted and should await the results of further ongoing clinical trials? [211]. In light of our processed food, depletion of minerals in the soil, our water and general bad eating habits make the taking of supplements very important.

\section{AN OUNCE OF PREVENTION}

Disease prevention is so important to our health care system. Prevention is "the elephant in the room" that has not really been addressed through active grant funding or in education at our medically oriented professional schools. Even the insurance companies have not encouraged in a meaningful way preventive medicine. The purpose of this section is to discuss the role of neutraceuticals in prevention of cancer, heart disease and immune suppression [212,213].

Cancer is one of the leading causes of death in the United States and around the world. Most modern drugtargeted therapies, besides being enormously expensive, are associated with serious side effects and morbidity. Still, the search continues for an ideal treatment that has minimal side effects and is cost-effective. Indeed, the design and development of chemopreventive agents that act on specific and/or multiple molecular and cellular targets is gaining support as a rational approach to prevent and treat cancer [25,213,214].

Recent nested case-control study and meta-analysis of numerous epidemiological studies show an inverse correlation between genistein intake and breast cancer risk. Furthermore, clinical studies in postmenopausal women support breast and uterine safety of purified naturally derived genistein administered for up to three years. In these studies, the preclinical and clinical evidence for the 
safety of natural Genistein has been shown [215].

The term "epigenetics" refers to modifications in gene expression caused by heritable, but potentially reversible, changes in DNA methylation and chromatin structure. Epigenetic alterations have been identified as promising new targets for cancer prevention strategies as they occur early during carcinogenesis and represent potentially initiating events for cancer development. Over the past few years, nutria-epigenetics - the influence of dietary components on mechanisms influencing the epigenome has emerged as an exciting new field in current epigenetic research.

Breast cancer is the second leading cause of cancerrelated deaths among women. Chemoprevention using Genistein and resveratrol for breast cancer may be a valid strategy [180]. Aberrant epigenetic alterations in the genome such as DNA methylation and chromatin remodeling play a significant role in breast cancer development. Since epigenetic alterations are considered to be more easily reversible compared to genetic changes, epigenetic therapy is potentially very useful in reversing some of these defects. Methylation of $\mathrm{CpG}$ islands is an important component of the epigenetic code, and a number of genes become abnormally methylated in breast cancer patients. Currently, several epigenetic-based synthetic drugs that can reduce DNA hypermethylation and histone deacetylation are undergoing preclinical and clinical trials. However, these chemicals are generally very toxic and do not have gene specificity. During carcinogenesis, major cellular functions and pathways, including drug metabolism, cell cycle regulation, potential to repair DNA damage or to induce apoptosis, response to inflammatory stimuli, cell signalling, and cell growth control and differentiation become deregulated. Recent evidence now indicates that epigenetic alterations contribute to these cellular defects, for example epigenetic silencing of detoxifying enzymes, tumor suppressor genes, cell cycle regulators, apoptosis-inducing and DNA repair genes, nuclear receptors, signal transducers and transcription factors by promoter methylation, and modifications of histones and non-histone proteins such as $\mathrm{p} 53, \mathrm{NF}-\kappa \mathrm{B}$, and the chaperone HSP90 by acetylation or methylation. Chemopreventive agents that target the epigenome include selenium genistein and curcumin, resveratrol that demonstrate the functional relevance of epigenetic mechanisms for health promoting or cancer preventive efficacy of natural products [216].

Potent dietary stem cell stimulate include Genistein, Resveratrol, piperine and vitamin D3 [217]. Cancer stem cells often have phenotypic and functional characteristics similar to normal stem cells including the properties of self-renewal and differentiation. Uncontrolled self-renewal may explain cancer relapses and may represent a critical target for cancer prevention. Dietary constituents such as vitamins genistein, resveratrol, curcumin, piperine have been shown to modify self-renewal properties of cancer stem cells. Response to food components does not appear to be tissue or organ specific but it suggests possibility of having common cellular mechanisms [217, 218].

Interest in natural product pharmacology has surged in the last 25 years and particularly risen at exponential rates since ten years. Phytochemicals curcumin, resveratrol and genistein are especially noteworthy in the literature [219].

Epidemiological studies have shown that Asian women are less prone to breast cancer due to their high consumption of soy food than the Caucasian women of western countries. Moreover, complementary/and or alternative medicines are commonly used by Asian populations, which use genistein, curcumin, and resveratrol. These bioactive components are able to modulate epigenetic events, and their epigenetic targets are known to be associated with breast cancer prevention and therapy. This approach could facilitate the discovery and development of novel drugs for the treatment of breast cancer. In this brief review, we will summarize the epigenetic events associated with breast cancer and the potential of some of these bioactive dietary components to modulate these events and thus, afford new therapeutic or preventive approaches [220].

Prostate cancer is the leading non-skin malignancy detected in US males and the second cause of death due to male cancer, in the US. Supplements, such as Genistein, that slow down the growth and progression of prostate cancer are potentially very effective in reducing the burden of prostate cancer, particularly if these treatments also prevent the development of new prostatic malignancies $[221,222]$.

The amount of micronized Resveratrol was retained in the liver and plasma 3.6 fold over non-micronized Resveratrol over single dose administration. Furthermore, capase-3, a marker for apoptosis, was significantly increased by $39 \%$ in malignant hepatic tissues following micronized Resveratrol treatment [223].

Curcumin is very important as a direct neutraceuticals agent against esophageal cancer [224]. Curcumin is used for the prevention and treatment of cardiovascular, diabetic and neurodegenerative diseases, as well as, cancer displaying amazing molecular versatility. Increasing evidence suggests mTOR is a master kinase, regulating cell growth/proliferation and survival and is a novel target of curcumin.

Proper management of tumorigenesis requires the development of multi-targeted therapies. Several adverse effects associated with present day cancer therapies is found and there is the absolute need for multi-targeted safe anticancer drug. Curcumin is just one example of 
blend of anti-carcinogenic, pro-apoptotic, anti-angiogenic, anti-metastatic, immunomodulatory and antioxidant activities [225-228].

Head and neck squamous cell carcinoma, as an example of the importance of prevention, is one of the most fatal cancers worldwide despite advances in its management. The overall survival for patients has not improved significantly due to advanced stages at diagnosis, high recurrence rate after surgical removal, and second primary tumor development. This cancer underscores the importance of novel strategies for cancer prevention. Many natural dietary compounds have been identified with multiple molecular targets, effective in the prevention and treatment of cancer [229].

The Mechanism of action of curcumin in hematologic and other malignancies is the inhibition of several cell signaling pathways at multiple levels, such as transcription factors (NF-B and AP-1), enzymes (COX-2, MMPs), cell cycle arrest (cyclin D1), proliferation (EGFR and Akt), survival pathways $(\beta$-catenin and adhesion molecules) and TNF. Curcumin up-regulates caspase family proteins and down regulates anti-apoptotic genes $(\mathrm{Bcl}-2$ and $\mathrm{Bcl}-\mathrm{X}(\mathrm{L}))$ [230].

Effects of trans-resveratrol on colon cancer has recently been reviewed [231,232]. Current evidence of the trans-Resveratrol not only reduces the number of preneoplastic lesions but also the incidence and multiplicity of tumors with clinical trial data $[223,233]$.

\section{ACKNOWLEDGEMENTS}

We thank our students, Hannah Rose, Weston Keen, Charlie Overturf, Mark Allen, Seth Blackwell and Patrick Kent for their assistance in researching for this manuscript. We thank Tony Kirk, Bonita Thornthwaite and Edra Shalla for their untiring rewriting of this manuscript. Special thanks to Kyle Thornthwaite for his animations.

\section{REFERENCES}

[1] Pietras, R.J. and Weinberg, O.K. (2005) Antiangiogenic steroids in human cancer therapy. Evidence-Based Complementary and Alternative Medicine, 2, 49-57. doi:10.1093/ecam/neh066

[2] Tascilar, M., et al. (2006) Complementary and alternative medicine during cancer treatment: Beyond innocence. The Oncologist, 11, 732-741. doi:10.1634/theoncologist.11-7-732

[3] Hagerty, R.G., et al. (2005) Communicating with realism and hope: Incurable cancer patients' views on the disclosure of prognosis. Journal of Clinical Oncology, 23, 1278-1288.

[4] Surh, Y.J. and Chun, K.S. (2007) Cancer chemopreventive effects of curcumin. Advances in Experimental Medicine and Biology, 595, 149-172. doi:10.1007/978-0-387-46401-5 5

[5] Chainani-Wu, N. (2003) Safety and anti-inflammatory activity of curcumin: A component of tumeric (Curcuma longa). The Journal of Alternative and Complementary Medicine, 9, 161-168. doi:10.1089/107555303321223035

[6] Duvoix, A., et al. (2005) Chemopreventive and therapeutic effects of curcumin. Cancer Letters, 223, 181-190. doi:10.1016/j.canlet.2004.09.041

[7] Hsu, C.H. and Cheng, A.L. (2007) Clinical studies with curcumin. Advances in Experimental Medicine and Biology, 595, 471-480. doi:10.1007/978-0-387-46401-5_21

[8] Bisht, M., Bist, S.S. and Dhasmana, D.C. (2010) Biological response modifiers: Current use and future prospects in cancer therapy. Indian Journal of Cancer, 47, 443-451. doi:10.4103/0019-509X.73559

[9] Johnson, J.J. and Mukhtar, H. (2007) Curcumin for chemoprevention of colon cancer. Cancer Letters, 255, 170181. doi:10.1016/j.canlet.2007.03.005

[10] Ferguson, L.R. and Philpott, M. (2007) Cancer prevention by dietary bioactive components that target the immune response. Current Cancer Drug Targets, 7, 459-464.

[11] Singh, S. and Khar, A. (2006) Biological effects of curcumin and its role in cancer chemoprevention and therapy. Anti-Cancer Agents in Medicinal Chemistry, 6, 259-270.

[12] Jagetia, G.C. and Aggarwal, B.B. (2007) "Spicing up" of the immune system by curcumin. Journal of Clinical Immunology, 27, 19-35. doi:10.1007/s10875-006-9066-7

[13] Sharma, R.A., Gescher, A.J. and Steward, W.P. (2005) Curcumin: The story so far. European Journal of Cancer, 41, 1955-1968. doi:10.1016/j.ejca.2005.05.009

[14] John, V.D., Kuttan, G. and Krishnankutty, K. (2002) Antitumour studies of metal chelates of synthetic curcumanoids. Journal of Experimental \& Clinical Cancer Research, 21, 219-224.

[15] Hadi, S.M., et al. (2000) Putative mechanism for anticancer and apoptosis-inducing properties of plant-derived polyphenolic compounds. IUBMB Life, 50, 167-171.

[16] Yoshino, M., et al. (2004) Prooxidant activity of curcumin: Copper-dependent formation of 8-hydroxy-2'-deoxyguanosine in DNA and induction of apoptotic cell death. Toxicology in Vitro, 18, 783-789. doi:10.1016/j.tiv.2004.03.009

[17] Thompson, K.H., et al. (2004) Complementary inhibition of synoviocyte, smooth muscle cell or mouse lymphoma cell proliferation by a vanadyl curcumin complex compared to curcumin alone. Journal of Inorganic Biochemistry, 98, 2063-2070.

doi:10.1016/j.jinorgbio.2004.09.011

[18] Garcea, G., et al. (2005) Consumption of the putative chemopreventive agent curcumin by cancer patients: Assessment of curcumin levels in the colorectum and their pharmacodynamic consequences. Cancer Epidemiology Biomarkers \& Prevention, 14,120-125.

[19] Adams, B.K., et al. (2004) Synthesis and biological evaluation of novel curcumin analogs as anti-cancer and anti-angiogenesis agents. Bioorganic \& Medicinal Chemistry, 12, 3871-3883. doi:10.1016/j.bmc.2004.05.006

[20] Sun, C.Y., et al. (2004) Experimental study on anticancer effect of curcumin on Raji cells in Vitro. Zhongguo Zhong Xi Yi Jie He Za Zhi, 24, 1003-1006. 
[21] Shao, Z.M., et al. (2002) Curcumin exerts multiple suppressive effects on human breast carcinoma cells. International Journal of Cancer, 98, 234-240. doi: $10.1002 /$ ijc. 10183

[22] Kim, J.H., et al. (2002) Microarray-based analysis of anti-angiogenic activity of demethoxycurcumin on human umbilical vein endothelial cells: Crucial involvement of the down-regulation of matrix metalloproteinase. Cancer Science, 93, 1378-1385. doi:10.1111/j.1349-7006.2002.tb01247.x

[23] Bemis, D.L., Katz, A.E. and Buttyan, R. (2006) Clinical trials of natural products as chemopreventive agents for prostate cancer. Expert Opinion on Investigational Drugs, 15, 1191-1200. doi:10.1517/13543784.15.10.1191

[24] Campbell, F.C. and Collett, G.P. (2005) Chemopreventive properties of curcumin. Future Oncology, 1, 405-414. doi:10.1517/14796694.1.3.405

[25] Shanmugam, M.K., Kannaiyan, R. and Sethi, G. (2011) Targeting cell signaling and apoptotic pathways by dietary agents: Role in the prevention and treatment of cancer. Nutrition and Cancer, 63, 161-173. doi:10.1080/01635581.2011.523502

[26] Su, S.J., et al., The novel targets for anti-angiogenesis of genistein on human cancer cells. Biochemical Pharmacology, 69, 307-318. doi:10.1016/j.bcp.2004.09.025

[27] Ravindranath, M.H., et al. (2004) Anticancer therapeutic potential of soy isoflavone, genistein. Advances in Experimental Medicine and Biology, 546, 121-165.

[28] Kyle, E., et al. (1997) Genistein-induced apoptosis of prostate cancer cells is preceded by a specific decrease in focal adhesion kinase activity. Molecular Pharmacology, 51, 193-200.

[29] Lazarevic, B., et al. (2012) The effects of short-term genistein intervention on prostate biomarker expression in patients with localised prostate cancer before radical prostatectomy.The British Journal of Nutrition, 108, 1-10.

[30] Lazarevic, B., et al. (2011) Efficacy and safety of shortterm genistein intervention in patients with localized prostate cancer prior to radical prostatectomy: A randomized, placebo-controlled, double-blind phase 2 clinical trial. Nutrition and Cancer, 63, 889-898. doi:10.1080/01635581.2011.582221

[31] Sasamura, H., et al. (2004) Antiproliferative and antiangiogenic activities of genistein in human renal cell carcinoma. Urology, 64, 389-393. doi:10.1016/j.urology.2004.03.045

[32] Hillman, G.G., et al. (2001) Genistein potentiates the radiation effect on prostate carcinoma cells. Clinical Cancer Research, 7, 382-390.

[33] de la Taille, A., et al. (2001) Cancer of the prostate: Influence of nutritional factors. A new nutritional approach. La Presse Médicale, 30, 561-564.

[34] Ghafar, M.A., et al. (2002) Regression of prostate cancer following administration of genistein combined polysaccharide (GCP), a nutritional supplement: A case report. The Journal of Alternative and Complementary Medicine, 8, 493-497. doi:10.1089/107555302760253694

[35] Davis, J.N., et al. (2000) Inhibition of prostate specific antigen expression by genistein in prostate cancer cells. International Journal of Oncology, 16, 1091-1097.

[36] Davis, J.N., Kucuk, O. and Sarkar, F.H. (2002) Expression of prostate-specific antigen is transcriptionally regulated by genistein in prostate cancer cells. Molecular Carcinogenesis, 34, 91-101. doi:10.1002/mc.10053

[37] Shen, J.C., et al. (2000) Low-dose genistein induces cyclin-dependent kinase inhibitors and $\mathrm{G}_{1}$ cell-cycle arrest in human prostate cancer cells. Molecular Carcinogenesis, 29, 92-102.

doi:10.1002/1098-2744(200010)29:2<92::AID-MC6>3.0. $\mathrm{CO} ; 2-\mathrm{Q}$

[38] Piao, M., et al. (2006) Inhibition of endothelial cell proliferation, in Vitro angiogenesis, and the down-regulation of cell adhesion-related genes by genistein. Combined with a cDNA microarray analysis. Endothelium, 13, 249266. doi:10.1080/10623320600903940

[39] Shichinohe, T., et al. (2001) Development of lentiviral vectors for antiangiogenic gene delivery. Cancer Gene Therapy, 8, 879-889. doi:10.1038/sj.cgt. 7700388

[40] Shao, Z.M., et al. (1998) Genistein exerts multiple suppressive effects on human breast carcinoma cells. Cancer Research, 58, 4851-4857.

[41] Li, Y. and Sarkar, F.H. (2002) Gene expression profiles of genistein-treated PC3 prostate cancer cells. The Journal of Nutrition, 132, 3623-3631.

[42] Li, Y. and Sarkar, F.H. (2002) Down-regulation of invasion and angiogenesis-related genes identified by cDNA microarray analysis of PC3 prostate cancer cells treated with genistein. Cancer Letters, 186, 157-164. doi:10.1016/S0304-3835(02)00349-X

[43] Sarkar, F.H. and Li, Y. (2002) Mechanisms of cancer chemoprevention by soy isoflavone genistein. Cancer and Metastasis Reviews, 21, 265-280. doi:10.1023/A:1021210910821

[44] Konstantakopoulos, N., et al. (2006) Changes in gene expressions elicited by physiological concentrations of genistein on human endometrial cancer cells. Molecular Carcinogenesis, 45, 752-763. doi:10.1002/mc. 20187

[45] Khoshyomn, S., et al. (2000) Synergistic action of genistein and cisplatin on growth inhibition and cytotoxicity of human medulloblastoma cells. Pediatric Neurosurgery, 33, 123-131. doi:10.1159/000028993

[46] Raynal, N.J., et al. (2008) Antileukemic activity of genistein, a major isoflavone present in soy products. Journal of Natural Products, 71, 3-7. doi:10.1021/np070230s

[47] Banerjee, S., et al. (2007) In Vitro and in Vivo molecular evidence of genistein action in augmenting the efficacy of cisplatin in pancreatic cancer. International Journal of Cancer, 120, 906-917. doi:10.1002/ijc.22332

[48] Dijkstra, S.C., et al. (2010) Biomarkers of dietary exposure are associated with lower risk of breast fibroadenomas in Chinese women. The Journal of Nutrition, 140, 1302-1310. doi:10.3945/jn.109.119727

[49] Vauzour, D., et al. (2007) Inhibition of cellular proliferation by the genistein metabolite 5,7,3',4'-tetrahydroxylisoflavone is mediated by DNA damage and activation of the ATR signalling pathway. Archives of Biochemistry 
and Biophysics, 468, 159-166. doi:10.1016/j.abb.2007.09.021

[50] Meeran, S.M. and Katiyar, S.K. (2008) Cell cycle control as a basis for cancer chemoprevention through dietary agents. Frontiers in Bioscience, 13, 2191-2202. doi: $10.2741 / 2834$

[51] Ohigashi, H. and Murakami, A. (2004) Cancer prevention with food factors: Alone and in combination. Biofactors, 22, 49-55. doi:10.1002/biof.5520220109

[52] Ramos, S. (2007) Effects of dietary flavonoids on apoptotic pathways related to cancer chemoprevention. The Journal of Nutritional Biochemistry, 18, 427-442. doi:10.1016/i.jnutbio.2006.11.004

[53] Sarkar, F.H., et al. (2006) The role of genistein and synthetic derivatives of isoflavone in cancer prevention and therapy. Mini Reviews in Medicinal Chemistry, 6, 401407.

[54] Perabo, F.G., et al. (2008) Soy isoflavone genistein in prevention and treatment of prostate cancer. Prostate Cancer and Prostatic Diseases, 11, 6-12. doi:10.1038/sj.pcan.4501000

[55] Nagata, Y., et al. (2007) Dietary isoflavones may protect against prostate cancer in Japanese men. The Journal of Nutrition, 137, 1974-1979.

[56] Heald, C.L., et al. (2006) Phyto-oestrogen intake in Scottish men: Use of serum to validate a self-administered food-frequency questionnaire in older men. European Journal of Clinical Nutrition, 60, 129-135. doi:10.1038/sj.ejen.1602277

[57] Kurahashi, N., et al. (2007) Soy product and isoflavone consumption in relation to prostate cancer in Japanese men. Cancer Epidemiology, Biomarkers \& Prevention, 16, 538-545. doi:10.1158/1055-9965.EPI-06-0517

[58] Ko, K.P., et al. (2010) Isoflavones from phytoestrogens and gastric cancer risk: A nested case-control study within the Korean Multicenter Cancer Cohort. Cancer Epidemiology, Biomarkers \& Prevention, 19, 1292-1300. doi:10.1158/1055-9965.EPI-09-1004

[59] Cho, J. and Kim, Y. (2002) Sharks: A potential source of antiangiogenic factors and tumor treatments. Marine Biotechnology, 4, 521-525. doi:10.1007/s10126-002-0064-3

[60] Williams, J.I., et al. (2001) Squalamine treatment of human tumors in nu/nu mice enhances platinum-based chemotherapies. Clinical Cancer Research, 7, 724-733.

[61] Bhargava, P., et al. (2001) A phase I and pharmacokinetic study of squalamine, a novel antiangiogenic agent, in patients with advanced cancers. Clinical Cancer Research, 7, 3912-3919.

[62] Herbst, R.S., et al. (2003) A phase I/IIA trial of continuous five-day infusion of squalamine lactate (MSI-1256F) plus carboplatin and paclitaxel in patients with advanced non-small cell lung cancer. Clinical Cancer Research, 9, 4108-4115.

[63] Hao, D., et al. (2003) A Phase I and pharmacokinetic study of squalamine, an aminosterol angiogenesis inhibittor. Clinical Cancer Research, 9, 2465-2471.

[64] Bayes, M., Rabasseda, X. and Prous, J.R. (2005) Gate- ways to clinical trials. Methods and Findings in Experimental and Clinical Pharmacology, 27, 331-372.

[65] Emerson, M.V. and Lauer, A.K. (2007) Emerging therapies for the treatment of neovascular age-related macular degeneration and diabetic macular edema. BioDrugs, 21, 245-257. doi:10.2165/00063030-200721040-00005

[66] Connolly, B., et al. (2006) Squalamine lactate for exudative age-related macular degeneration. Ophthalmology Clinics of North America, 19, 381-391.

[67] Michels, S., Schmidt-Erfurth, U. and Rosenfeld, P.J. (2006) Promising new treatments for neovascular age-related macular degeneration. Expert Opinion on Investigational Drugs, 15, 779-793. doi: 10.1517/13543784.15.7.779

[68] Shepherd, F.A. and Sridhar, S.S. (2003) Angiogenesis inhibitors under study for the treatment of lung cancer. Lung Cancer, 41, 63-72. doi:10.1016/S0169-5002(03)00144-2

[69] Li, D., Williams, J.I. and Pietras, R.J. (2002) Squalamine and cisplatin block angiogenesis and growth of human ovarian cancer cells with or without HER-2 gene overexpression. Oncogene, 21, 2805-2814. doi:10.1038/sj.onc. 1205410

[70] Akhter, S., et al. (1999) Squalamine, a novel cationic steroid, specifically inhibits the brush-border $\mathrm{Na}^{+} / \mathrm{H}^{+}$exchanger isoform NHE3. American Journal of Physiology -Cell Physiology, 276, C136-C144.

[71] Teicher, B.A., et al. (1998) Potential of the aminosterol, squalamine in combination therapy in the rat 13,762 mammary carcinoma and the murine Lewis lung carcinoma. Anticancer Research, 18, 2567-2573.

[72] Paley, P.J., et al. (1997) Vascular endothelial growth factor expression in early stage ovarian carcinoma. Cancer, 80, 98-106. doi:10.1002/(SICI)1097-0142(19970701)80:1<98::AIDCNCR13>3.0.CO;2-A

[73] Yamamoto, S., et al. (1997) Expression of vascular endothelial growth factor (VEGF) in epithelial ovarian neoplasms: Correlation with clinicopathology and patient survival, and analysis of serum VEGF levels. British Journal of Cancer, 76, 1221-1227. doi:10.1038/bjc. 1997.537

[74] Alvarez, A.A., et al. (1999) The prognostic significance of angiogenesis in epithelial ovarian carcinoma. Clinical Cancer Research, 5, 587-591.

[75] Brunel, J.M., et al. (2005) Squalamine: A polyvalent drug of the future? Current Cancer Drug Targets, 5, 267-272.

[76] Havre, P.A., et al. (2002) Transformed and tumor-derived human cells exhibit preferential sensitivity to the thiol antioxidants, N-acetyl cysteine and penicillamine. Cancer Research, 62, 1443-1449.

[77] Alonso, A., et al. (2004) Prevention of radiocontrast nephropathy with $\mathrm{N}$-acetylcysteine in patients with chronic kidney disease: A meta-analysis of randomized, controlled trials. American Journal of Kidney Diseases, 43, 1-9.

[78] Agarwal, A., et al. (2004) N-acetyl-cysteine promotes angiostatin production and vascular collapse in an or- 
thotopic model of breast cancer. The American Journal of Pathology, 164, 1683-1696. doi:10.1016/S0002-9440(10)63727-3

[79] Albini, A., et al. (2001) Inhibition of angiogenesis-driven Kaposi's sarcoma tumor growth in nude mice by oral N-acetylcysteine. Cancer Research, 61, 8171-8178.

[80] Choi, C.H., et al. (2007) Phase II study of neoadjuvant chemotherapy with mitomycin-c, vincristine and cisplatin (MVC) in patients with stages IB2-IIB cervical carcinoma. Gynecologic Oncology, 104, 64-69. doi:10.1016/j.ygyno.2006.07.006

[81] Betten, D.P., et al. (2007) A prospective evaluation of shortened course oral $\mathrm{N}$-acetylcysteine for the treatment of acute acetaminophen poisoning. Annals of Emergency Medicine, 50, 272-279.

doi:10.1016/j.annemergmed.2006.11.010

[82] Paterson, R.L., Galley, H.F. and Webster, N.R. (2003) The effect of $\mathrm{N}$-acetylcysteine on nuclear factor-kappa $\mathrm{B}$ activation, interleukin-6, interleukin-8, and intercellular adhesion molecule-1 expression in patients with sepsis. Critical Care Medicine, 31, 2574-2578. doi:10.1097/01.CCM.0000089945.69588.18

[83] Uwe, S. (2008) Anti-inflammatory interventions of NFkappaB signaling: Potential applications and risks. Biochemical Pharmacology, 75, 1567-1579. doi:10.1016/j.bcp.2007.10.027

[84] Vecchiarelli, A., et al. (1994) Macrophage activation by $\mathrm{N}$-acetyl-cysteine in COPD patients. Chest, 105, 806-811.

[85] Mantovani, G., et al. (2003) Subcutaneous interleukin-2 in combination with medroxyprogesterone acetate and antioxidants in advanced cancer responders to previous chemotherapy: Phase II study evaluating clinical, quality of life, and laboratory parameters. Journal of Experimental Therapeutics and Oncology, 3, 205-219. doi:10.1046/j.1359-4117.2003.01096.x

[86] Pendyala, L. and Creaven, P.J. (1995) Pharmacokinetic and pharmacodynamic studies of $\mathrm{N}$-acetylcysteine, a potential chemopreventive agent during a phase I trial. Cancer Epidemiology, Biomarkers \& Prevention, 4, 245251.

[87] Wolchok, J.D., et al. (2003) Phase I trial of high dose paracetamol and carmustine in patients with metastatic melanoma. Melanoma Research, 13, 189-196. doi:10.1097/00008390-200304000-00013

[88] Haase, M., et al. (2007) Phase II, randomized, controlled trial of high-dose $\mathrm{N}$-acetylcysteine in high-risk cardiac surgery patients. Critical Care Medicine, 35, 1324-1331.

[89] Mantovani, G., et al. (2002) Phase II study of subcutaneously administered interleukin-2 in combination with medroxyprogesterone acetate and antioxidant agents as maintenance treatment in advanced cancer responders to previous chemotherapy. Oncology Reports, 9, 887-896.

[90] Zoran, D.L., et al. (1997) Wheat bran diet reduces tumor incidence in a rat model of colon cancer independent of effects on distal luminal butyrate concentrations. The Journal of Nutrition, 127, 2217-2225.

[91] Reddy, B.S., et al. (2000) Preventive potential of wheat bran fractions against experimental colon carcinogenesis:
Implications for human colon cancer prevention. Cancer Research, 60, 4792-4797.

[92] Martinez, M.E., et al. (1998) Design and baseline characteristics of study participants in the wheat bran fiber trial. Cancer Epidemiology, Biomarkers \& Prevention, 7, 813-816.

[93] Compher, C.W., et al. (1999) Wheat bran decreases aberrant crypt foci, preserves normal proliferation, and increases intraluminal butyrate levels in experimental colon cancer. Journal of Parenteral Enteral Nutrition, 23, 269277.

[94] Folino, M., McIntyre, A. and Young, G.P. (1995) Dietary fibers differ in their effects on large bowel epithelial proliferation and fecal fermentation-dependent events in rats. The Journal of Nutrition, 125, 1521-1528.

[95] Lupton, J.R. and Kurtz, P.P. (1993) Relationship of colonic luminal short-chain fatty acids and $\mathrm{pH}$ to in Vivo cell proliferation in rats. The Journal of Nutrition, 123, 15221530 .

[96] Zhang, J. and Lupton, J.R. (1994) Dietary fibers stimulate colonic cell proliferation by different mechanisms at different sites. Nutrition and Cancer, 22, 267-276. doi:10.1080/01635589409514352

[97] McIntyre, A., et al. (1991) Different fibers have different regional effects on luminal contents of rat colon. Gastroenterology, 101, 1274-1281.

[98] McIntyre, A., Gibson, P.R. and Young, G.P. (1993) Butyrate production from dietary fibre and protection against large bowel cancer in a rat model. Gut, 34, 386391. doi:10.1136/gut.34.3.386

[99] Newmark, H.L. and Lupton, J.R. (1990) Determinants and consequences of colonic luminal $\mathrm{pH}$ : Implications for colon cancer. Nutrition and Cancer, 14, 161-173. doi:10.1080/01635589009514091

[100] Kestell, P., et al. (1999) Studies on the mechanism of cancer protection by wheat bran: Effects on the absorption, metabolism and excretion of the food carcinogen 2-amino-3-methylimidazo[4,5-f]quinoline (IQ). Carcinogenesis, 20, 2253-2260. doi:10.1093/carcin/20.12.2253

[101] Ghoneum, M. and Matsuura, M. (2004) Augmentation of macrophage phagocytosis by modified arabinoxylan rice bran (MGN-3/biobran). International Journal of Immunopathology and Pharmacology, 17, 283-292.

[102] Chang, H., et al. (2004) Prognostic relevance of immunophenotyping in 379 patients with acute myeloid leukemia. Leukemia Research, 28, 43-48. doi:10.1016/S0145-2126(03)00180-2

[103] Lee, C.Y. and Wan, J.M. (2002) Immunoregulatory and antioxidant performance of alpha-tocopherol and selenium on human lymphocytes. Biological Trace Element Research, 86, 123-136. doi:10.1385/BTER:86:2:123

[104] Muralikrishna, G. and Rao, M.V. (2007) Cereal non-cellulosic polysaccharides: Structure and function relationship-An overview. Critical Reviews in Food Science and Nutrition, 47, 599-610. doi:10.1080/10408390600919056

[105] Glei, M., et al. (2006) Both wheat (Triticum aestivum) bran arabinoxylans and gut flora-mediated fermentation 
products protect human colon cells from genotoxic activities of 4-hydroxynonenal and hydrogen peroxide. Journal of Agricultural and Food Chemistry, 54, 20882095. doi:10.1021/if052768e

[106] Ferreira, I.C., et al. (2010) Compounds from wild mushrooms with antitumor potential. Anti-Cancer Agents in Medicinal Chemistry, 10, 424-436.

[107] Wasser, S.P. and Weis, A.L. (1999) Therapeutic effects of substances occurring in higher basidiomycetes mushrooms: A modern perspective. Critical Reviews in Immunology, 19, 65-96.

[108] Chihara, G. (1992) Recent progress in immunopharmacology and therapeutic effects of polysaccharides. Developments in Biological Standardization, 77, 191-197.

[109] Ooi, V.E. and Liu, F. (2000) Immunomodulation and anti-cancer activity of polysaccharide-protein complexes. Current Medicinal Chemistry, 7, 715-729.

[110] Borchers, A.T., et al. (1999) Mushrooms, tumors, and immunity. Proceedings of the Society for Experimental Biology and Medicine, 221, 281-293. doi:10.1046/j.1525-1373.1999.d01-86.x

[111] Jong, S.C., Birmingham, J.M. and Pai, S.H. (1991) Immunomodulatory substances of fungal origin. Journal of Immunology and Immunopharmacology, 9, 115-122.

[112] Hobbs, C.R. (2000) Medicinal value of Lentinus edodes (Berk.) Sing. (Agaricomycetideae). A literature review. International Journal of Medicinal Mushrooms, 2, 287302.

[113] Taguchi, T., et al. (1985) Results of phase III study of lentinan. Gan to Kagaku Ryoho, 12, 366-378.

[114] Oba, K., et al. (2009) Individual patient based metaanalysis of lentinan for unresectable/recurrent gastric cancer. Anticancer Research, 29, 2739-2745.

[115] Hazama, S., et al. (2009) Efficacy of orally administered superfine dispersed lentinan (beta-1,3-glucan) for the treatment of advanced colorectal cancer. Anticancer Research, 29, 2611-2617.

[116] Ross, G.D., et al. (1999) Therapeutic intervention with complement and beta-glucan in cancer. Immunopharmacology, 42, 61-74. doi:10.1016/S0162-3109(99)00013-2

[117] Kerekgyarto, C., et al. (1996) Strain differences in the cytotoxic activity and TNF production of murine macrophages stimulated by lentinan. International Journal of Immunopharmacology, 18, 347-353. doi:10.1016/S0192-0561(96)00038-0

[118] Yoshino, S., et al. (2010) Improvement of QOL and prognosis by treatment of superfine dispersed lentinan in patients with advanced gastric cancer. Hepatogastroenterology, 57, 172-177.

[119] Kataoka, H., et al. (2009) Lentinan with S-1 and paclitaxel for gastric cancer chemotherapy improve patient quality of life. Hepatogastroenterology, 56, 547-550.

[120] Wang, J.L., et al. (2012) Combination therapy with lentinan improves outcomes in patients with esophageal carcinoma. Molecular Medicine Report, 5, 745-748.

[121] Vanotti, A., et al. (2007) Overview on pathophysiology and newer approaches to treatment of peripheral neu- ropathies. CNS Drugs, 21, 3-12. doi:10.2165/00023210-200721001-00002

[122] D. De Grandis, (2007) Acetyl-1-carnitine for the treatment of chemotherapy-induced peripheral neuropathy: A short review. CNS Drugs, 21, 39-43. doi:10.2165/00023210-200721001-00006

[123] Youle, M. (2007) Acetyl-1-carnitine in HIV-associated antiretroviral toxic neuropathy. CNS Drugs, 21, 25-30. doi:10.2165/00023210-200721001-00004

[124] Sima, A.A. (2007) Acetyl-1-carnitine in diabetic polyneuropathy: Experimental and clinical data. CNS Drugs, 21, 13-23. doi:10.2165/00023210-200721001-00003

[125] Youle, M. and Osio, M. (2007) A double-blind, parallel-group, placebo-controlled, multicentre study of acetyl1-carnitine in the symptomatic treatment of antiretroviral toxic neuropathy in patients with HIV-1 infection. HIV Medicine, 8, 241-250. doi:10.1111/j.1468-1293.2007.00467.x

[126] Murosaki, S., et al. (2007) A combination of caffeine, arginine, soy isoflavones, and L-carnitine enhances both lipolysis and fatty acid oxidation in 3T3-L1 and HepG2 cells in Vitro and in KK mice in Vivo. The Journal of $\mathrm{Nu}$ trition, 137, 2252-2257.

[127] Carroll, J.K., et al. (2007) Pharmacologic treatment of cancer-related fatigue. The Oncologist, 12, 43-51. doi:10.1634/theoncologist.12-S1-43

[128] Gramignano, G., et al. (2006) Efficacy of 1-carnitine administration on fatigue, nutritional status, oxidative stress, and related quality of life in 12 advanced cancer patients undergoing anticancer therapy. Nutrition, 22, 136-145. doi:10.1016/j.nut.2005.06.003

[129] Cruciani, R.A., et al. (2006) Safety, tolerability and symptom outcomes associated with L-carnitine supplementation in patients with cancer, fatigue, and carnitine deficiency: A phase I/II study. Journal of Pain and Symptom Management, 32, 551-559. doi:10.1016/j.jpainsymman.2006.09.001

[130] Sood, A., et al. (2007) A critical review of complementtary therapies for cancer-related fatigue. Integrative Cancer Therapies, 6, 8-13. doi:10.1177/1534735406298143

[131] Delaney, C.E., Hopkins, S.P. and Addison, C.L. (2007) Supplementation with 1-carnitine does not reduce the efficacy of epirubicin treatment in breast cancer cells. Cancer Letters, 252, 195-207. doi:10.1016/j.canlet.2006.12.027

[132] Dong, M.H. and Kaunitz, J.D. (2006) Gastroduodenal mucosal defense. Current Opinion in Gastroenterology, 22, 599-606. doi:10.1097/01.mog.0000245540.87784.75

[133] Lebrun, C., et al. (2006) Levocarnitine administration in multiple sclerosis patients with immunosuppressive therapy-induced fatigue. Multiple Sclerosis Journals, 12, 321-324. doi:10.1191/135248506ms1275oa

[134] Mantovani, G., et al. (2003) Antioxidant agents are effective in inducing lymphocyte progression through cell cycle in advanced cancer patients: Assessment of the most important laboratory indexes of cachexia and oxidative stress. Journal of Molecular Medicine, 81, 664-673. doi:10.1007/s00109-003-0476-1 
[135] Weitsman, G.E., et al. (2003) Vitamin D enhances caspase-dependent and independent TNF-induced breast cancer cell death: The role of reactive oxygen species. Annals of New York Academy of Sciences, 1010, 437-440.

[136] Mantovani, G., et al. (2003) The impact of different antioxidant agents alone or in combination on reactive oxygen species, antioxidant enzymes and cytokines in a series of advanced cancer patients at different sites: Correlation with disease progression. Free Radical Research, 37, 213-223. doi:10.1080/10715760303849

[137] Zahid, M., et al. (2007) Inhibition of depurinating estrogen-DNA adduct formation by natural compounds. Chemical Research in Toxicology, 20, 1947-1953. doi:10.1021/tx700269s

[138] Novotny, L., Rauko, P. and Cojocel, C. (2008) Alphalipoic acid: The potential for use in cancer therapy. Neoplasma, 55, 81-86.

[139] Rock, E. and DeMichele, A. (2003) Nutritional approaches to late toxicities of adjuvant chemotherapy in breast cancer survivors. The Journal of Nutrition, 133, 3785S-3793S.

[140] Schwartz, L., et al. (2012) Tumor regression with a combination of drugs interfering with the tumor metabolism: Efficacy of hydroxycitrate, lipoic acid and capsaicin. Investigational New Drugs, 31, 256-264.

[141] Moungjaroen, J., et al. (2006) Reactive oxygen species mediate caspase activation and apoptosis induced by lipoic acid in human lung epithelial cancer cells through Bcl-2 down-regulation. The Journal of Pharmacology and Experimental Therapeutics, 319, 1062-1069. doi:10.1124/jpet.106.110965

[142] Miquel, J., et al. (2006) Menopause: A review on the role of oxygen stress and favorable effects of dietary antioxidants. Archives of Gerontology and Geriatrics, 42, 289306. doi:10.1016/j.archger.2005.08.005

[143] Valko, M., et al. (2006) Free radicals, metals and antioxidants in oxidative stress-induced cancer. Chemico-Biological Interactions, 160, 1-40. doi:10.1016/j.cbi.2005.12.009

[144] Ratnam, D.V., et al. (2006) Role of antioxidants in prophylaxis and therapy: A pharmaceutical perspective. Journal of Controlled Release, 113, 189-207. doi:10.1016/j.jconrel.2006.04.015

[145] Matkovics, A. (2006) Recent changes in concepts of antioxidant treatment. Orvosi Hetilap, 147, 747-752.

[146] Schmelzer, C., et al. (2007) Functional connections and pathways of coenzyme Q10-inducible genes: An in-silico study. IUBMB Life, 59, 628-633. doi:10.1080/15216540701545991

[147] Pepe, S., et al. (2007) Coenzyme $Q_{10}$ in cardiovascular disease. Mitochondrion, 7, S154-S167. doi:10.1016/j.mito.2007.02.005

[148] Rosenfeldt, F., et al. (2005) Coenzyme $\mathrm{Q}_{10}$ therapy before cardiac surgery improves mitochondrial function and in Vitro contractility of myocardial tissue. The Journal of Thoracic and Cardiovascular Surgery, 129, 25-32. doi:10.1016/j.jtcvs.2004.03.034

[149] Bailey, D.M., et al. (2007) Electron paramagnetic spec- troscopic evidence of exercise-induced free radical accumulation in human skeletal muscle. Free Radical Research, 41, 182-190. doi:10.1080/10715760601028867

[150] Thomas, J.E., Lee, N. and Thompson, P.D. (2007) Statins provoking MELAS syndrome. A case report. European Neurology, 57, 232-235. doi:10.1159/000101287

[151] Langsjoen, P.H., et al. (2005) Treatment of statin adverse effects with supplemental Coenzyme $\mathrm{Q}_{10}$ and statin drug discontinuation. Biofactors, 25, 147-152. doi:10.1002/biof.5520250116

[152] Lamperti, C., et al. (2005) Muscle coenzyme $\mathrm{Q}_{10}$ level in statin-related myopathy. Archives of Neurology, 62, 17091712. doi:10.1001/archneur.62.11.1709

[153] Stocker, R., et al. (2006) Neither plasma coenzyme $Q_{10}$ concentration, nor its decline during pravastatin therapy, is linked to recurrent cardiovascular disease events: A prospective case-control study from the LIPID study. Atherosclerosis, 187, 198-204. doi:10.1016/j.atherosclerosis.2005.09.004

[154] Siemieniuk, E. and Skrzydlewska, E. (2005) Coenzyme $\mathrm{Q}_{10}$ : Its biosynthesis and biological significance in animal organisms and in humans. Postepy Higieny I Medycyny Doświadczalnej (Online), 59, 150-159.

[155] Tavintharan, S., et al. (2007) Reduced mitochondrial coenzyme $\mathrm{Q}_{10}$ levels in HepG2 cells treated with highdose simvastatin: A possible role in statin-induced heaptotoxicity? Toxicology and Applied Pharmacology, 223, 173-179. doi:10.1016/j.taap.2007.05.013

[156] McCarthy, S., et al. (2004) Paraquat induces oxidative stress and neuronal cell death; neuroprotection by water-soluble Coenzyme $\mathrm{Q}_{10}$. Toxicology and Applied Pharmacology, 201, 21-31. doi:10.1016/j.taap.2004.04.019

[157] Korkina, L., et al. (2003) Coenzyme $\mathrm{Q}_{10}$-containing composition (Immugen) protects against occupational and environmental stress in workers of the gas and oil Industry. Biofactors, 18, 245-254. doi:10.1002/biof.5520180227

[158] Bruge, F., et al. (2003) Effect of UV-C mediated oxidative stress in leukemia cell lines and its relation to ubiquinone content. Biofactors, 18, 51-63. doi:10.1002/biof.5520180207

[159] Ebadi, M., et al. (2004) Coenzyme $Q_{10}$ inhibits mitochondrial complex-1 down-regulation and nuclear factor-kappa B activation. Journal of Cellular and Molecular Medicine, 8, 213-222. doi:10.1111/j.1582-4934.2004.tb00276.x

[160] Hyun, D.H., et al. (2006) Calorie restriction up-regulates the plasma membrane redox system in brain cells and suppresses oxidative stress during aging. Proceedings of the National Academy of Sciences of the United States of America, 103, 19908-19912. doi:10.1073/pnas.0608008103

[161] Bhagavan, H.N., et al. (2007) Assessment of coenzyme $\mathrm{Q}_{10}$ absorption using an in Vitro digestion-Caco-2 cell model. International Journal of Pharmaceutics, 333, 112-117. doi:10.1016/j.ijpharm.2006.10.007

[162] Niklowitz, P., et al. (2007) Coenzyme $Q_{10}$ concentration in the plasma of children suffering from acute lymphoastic leukaemia before and during induction treatment. 
Biofactors, 29, 83-89. doi:10.1002/biof.552029208

[163] van Dalen, E.C., Caron, H.N. and Kremer, L.C. (2007) Prevention of anthracycline-induced cardiotoxicity in children: The evidence. European Journal of Cancer, 43, 1134-1140. doi:10.1016/j.ejca.2007.01.040

[164] Conklin, K.A. (2005) Coenzyme $Q_{10}$ for prevention of anthracycline-induced cardiotoxicity. Integrative Cancer Therapies, 4, 110-130. doi:10.1177/1534735405276191

[165] Bryant, J., et al. (2007) Cardioprotection against the toxic effects of anthracyclines given to children with cancer: A systematic review. Health Technology Assessment, 11, iii, ix-x, 1-84.

[166] Premkumar, V.G., et al. (2007) Effect of coenzyme $Q_{10}$, riboflavin and niacin on serum CEA and CA 15-3 levels in breast cancer patients undergoing tamoxifen therapy. Biological \& Pharmaceutical Bulletin, 30, 367-370. doi:10.1248/bpb.30.367

[167] Yuvaraj, S., et al. (2007) Ameliorating effect of coenzyme $\mathrm{Q}_{10}$, riboflavin and niacin in tamoxifen-treated postmenopausal breast cancer patients with special reference to lipids and lipoproteins. Clinical Biochemistry, 40, 623628. doi:10.1016/j.clinbiochem.2007.02.003

[168] Palan, P.R., et al. (2005) Effects of menopause and hormone replacement therapy on serum levels of coenzyme Q10 and other lipid-soluble antioxidants. Biofactors, 25, 61-66. doi:10.1002/biof.5520250107

[169] Rusciani, L., et al. (2007) Recombinant interferon alpha-2b and coenzyme $\mathrm{Q}_{10}$ as a postsurgical adjuvant therapy for melanoma: A 3-year trial with recombinant interferon-alpha and 5-year follow-up. Melanoma Research, 17, 177-183. doi:10.1097/CMR.0b013e32818867a0

[170] Rusciani, L., et al. (2006) Low plasma coenzyme $\mathrm{Q}_{10}$ levels as an independent prognostic factor for melanoma progression. Journal of the American Academy of Dermatology, 54, 234-241. doi:10.1016/i.jaad.2005.08.031

[171] Brea-Calvo, G., et al. (2006) Chemotherapy induces an increase in coenzyme $\mathrm{Q}_{10}$ levels in cancer cell lines. Free Radical Biology and Medicine, 40, 1293-1302. doi:10.1016/j.freeradbiomed.2005.11.014

[172] Roffe, L., Schmidt, K. and Ernst, E. (2004) Efficacy of coenzyme $\mathrm{Q}_{10}$ for improved tolerability of cancer treatments: A systematic review. Journal of Clinical Oncology, 22, 4418-4424.

[173] Fulda, S. and Debatin, K.M. (2006) Resveratrol modulation of signal transduction in apoptosis and cell survival: A mini-review. Cancer Detection and Prevention, 30, 217-223. doi:10.1016/j.cdp.2006.03.007

[174] Vinod, B.S., Maliekal, T.T. and Anto, R.J. (2012) Phytochemicals as chemosensitizers: From molecular mechanism to clinical significance. Antioxidants \& Redox Signaling, 18, 1307-1348.

[175] Hull, M.A. (2012) Nutritional agents with anti-lnflammatory properties in chemoprevention of colorectal neoplasia. Recent Results in Cancer Research, 191, 143-156. doi:10.1007/978-3-642-30331-9 8

[176] Athar, M., et al. (2007) Resveratrol: A review of preclinical studies for human cancer prevention. Toxicology and Applied Pharmacology, 224, 274-283. doi:10.1016/j.taap.2006.12.025

[177] Leon-Galicia, I., et al. (2012) Resveratrol induces downregulation of DNA repair genes in MCF-7 human breast cancer cells. European Journal Cancer Prevention, 22, 11-20.

[178] Kim, Y.S., Sull, J.W. and Sung, H.J. (2012) Suppressing effect of resveratrol on the migration and invasion of human metastatic lung and cervical cancer cells. Molecular Biology Reports, 39, 8709-8716. doi:10.1007/s11033-012-1728-3

[179] Scoditti, E., et al. (2012) Mediterranean diet polyphenols reduce inflammatory angiogenesis through MMP-9 and COX-2 inhibition in human vascular endothelial cells: A potentially protective mechanism in atherosclerotic vascular disease and cancer. Archives of Biochemistry and Biophysics, 527, 81-89.doi:10.1016/j.abb.2012.05.003

[180] Liu, M.M., Huang, Y. and Wang, J. (2012) Developing phytoestrogens for breast cancer prevention. Anti-Cancer Agents in Medicinal Chemistry, 12, 1306-1313.

[181] Shankar, S., Singh, G. and Srivastava, R.K. (2007) Chemoprevention by resveratrol: Molecular mechanisms and therapeutic potential. Frontiers in Bioscience, 12, 48394854. doi: $10.2741 / 2432$

[182] Boocock, D.J., et al. (2007) Phase I dose escalation pharmacokinetic study in healthy volunteers of resveratrol, a potential cancer chemopreventive agent. Cancer Epidemiology, Biomarkers \& Prevention, 16, 1246-1252. doi:10.1158/1055-9965.EPI-07-0022

[183] Delmas, D., et al. (2006) Resveratrol as a chemopreventive agent: A promising molecule for fighting cancer. Current Drug Targets, 7, 423-442.

[184] Aziz, M.H., et al. (2005) Chemoprevention of skin cancer by grape constituent resveratrol: Relevance to human disease? FASEB Journal, 19, 1193-1195.

[185] Stojanovic, S., Sprinz, H. and Brede, O. (2001) Efficiency and mechanism of the antioxidant action of trans-resveratrol and its analogues in the radical liposome oxidation. Archives of Biochemistry and Biophysics, 391, 79-89. doi:10.1006/abbi.2001.2388

[186] Brito, P., Almeida, L.M. and Dinis, T.C. (2002) The interaction of resveratrol with ferrylmyoglobin and peroxynitrite; Protection against LDL oxidation. Free Radical Research, 36, 621-631. doi:10.1080/10715760290029083

[187] Bradamante, S., Barenghi, L. and Villa, A. (2004) Cardiovascular protective effects of resveratrol. Cardiovascular Drug Reviews, 22, 169-188. doi:10.1111/j.1527-3466.2004.tb00139.x

[188] Das, S. and Das, D.K. (2007) Anti-inflammatory responses of resveratrol. Inflammation \& Allergy Drug Targets, 6, 168-173. doi:10.2174/187152807781696464

[189] Goswami, S.K. and Das, D.K. (2009) Resveratrol and chemoprevention. Cancer Letters, 284, 1-6. doi:10.1016/j.canlet.2009.01.041

[190] Aggarwal, B.B., et al. (2004) Role of resveratrol in prevention and therapy of cancer: Preclinical and clinical studies. Anticancer Research, 24, 2783-2840. 
[191] Gescher, A.J. and Steward, W.P. (2003) Relationship between mechanisms, bioavailibility, and preclinical chemopreventive efficacy of resveratrol: A conundrum. Cancer Epidemiology, Biomarkers \& Prevention, 12, 953957.

[192] Bass, T.M., et al. (2007) Effects of resveratrol on lifespan in Drosophila melanogaster and Caenorhabditis elegans. Mechanisms of Ageing and Development, 128, 546-552. doi:10.1016/j.mad.2007.07.007

[193] Bartel, J., et al. (2007) Activity of the glutathione peroxidase-2. Differences in the selenium-dependent expression between colon and small intestine. Cancer Genomics \& Proteomics, 4, 369-372.

[194] Steinbrenner, H., et al. (2007) Post-translational processing of selenoprotein P: Implications of glycosylation for its utilisation by target cells. Biological Chemistry, 388, 1043-1051. doi:10.1515/BC.2007.136

[195] Philchenkov, A., et al. (2007) Comparative analysis of apoptosis induction by selenium compounds in human lymphoblastic leukemia MT-4 cells. Experimental Oncology, 29, 257-261.

[196] Meplan, C. and Hesketh, J. (2012) The influence of selenium and selenoprotein gene variants on colorectal cancer risk. Mutagenesis, 27, 177-186. doi:10.1093/mutage/ger058

[197] Bardia, A., et al. (2008) Efficacy of antioxidant supplementation in reducing primary cancer incidence and mortality: Systematic review and meta-analysis. Mayo Clinic Proceedings, 83, 23-34. doi:10.4065/83.1.23

[198] Li, S., et al. (2007) Doxorubicin and selenium cooperatively induce fas signaling in the absence of Fas/Fas ligand interaction. Anticancer Research, 27, 3075-3082.

[199] Santos, R.A. and Takahashi, C.S. (2008) Anticlastogenic and antigenotoxic effects of selenomethionine on doxorubicin-induced damage in Vitro in human lymphocytes. Food and Chemical Toxicology, 46, 671-677. doi:10.1016/j.fct.2007.09.090

[200] Cheung, E., et al. (2008) Diet and prostate cancer risk reduction. Expert Review of Anticancer Therapy, 8, 43-50. doi:10.1586/14737140.8.1.43

[201] Juliger, S., et al. (2007) Chemosensitization of B-cell lymphomas by methylseleninic acid involves nuclear factor-kappaB inhibition and the rapid generation of other selenium species. Cancer Research, 67, 10984-10992. doi:10.1158/0008-5472.CAN-07-0519

[202] Murawaki, Y., et al. (2008) Aberrant expression of selenoproteins in the progression of colorectal cancer. Cancer Letters, 259, 218-230. doi:10.1016/j.canlet.2007.10.019

[203] Ma, J.L., et al. (2012) Fifteen-year effects of Helicobacter pylori, garlic, and vitamin treatments on gastric cancer incidence and mortality. Journal of the National Cancer Institiute, 104, 488-492. doi:10.1093/jnci/djs003

[204] Chen, K.M., et al. (2007) Inhibition of nuclear factorkappaB DNA binding by organoselenocyanates through covalent modification of the p50 subunit. Cancer Research, 67, 10475-10483.

doi:10.1158/0008-5472.CAN-07-2510
[205] Lee, E.H., et al. (2011) Effects of selenium supplements on cancer prevention: Meta-analysis of randomized controlled trials. Nutrition and Cancer, 63, 1185-1195. doi:10.1080/01635581.2011.607544

[206] Sanmartin, C., et al. (2011) Selenium and clinical trials: New therapeutic evidence for multiple diseases. Current Medicinal Chemistry, 18, 4635-4650.

[207] Cuello, S., et al. (2007) Selenium methylselenocysteine protects human hepatoma HepG2 cells against oxidative stress induced by tert-butyl hydroperoxide. Analytical and Bioanalytical Chemistry, 389, 2167-2178. doi:10.1007/s00216-007-1626-Z

[208] Schmutzler, C., et al. (2007) Selenoproteins of the thyroid gland: Expression, localization and possible function of glutathione peroxidase 3. Biological Chemistry, 388, 1053-1059. doi:10.1515/BC.2007.122

[209] Yanagisawa, H. (2008) Zinc deficiency and clinical practice-Validity of zinc preparations. Yakugaku Zasshi, 128, 333-339. doi:10.1248/yakushi.128.333

[210] Yan, M., et al. (2008) Zinc deficiency alters DNA damage response genes in normal human prostate epithelial cells. The Journal of Nutrition, 138, 667-673.

[211] Lonn, E.M. and Yusuf, S. (1997) Is there a role for antioxidant vitamins in the prevention of cardiovascular diseases? An update on epidemiological and clinical trials data. The Canadian Journal of Cardiology, 13, 957-965.

[212] Bansal, S.S., et al. (2011) Advanced drug delivery systems of curcumin for cancer chemoprevention. Cancer Prevention Research, 4, 1158-1171. doi:10.1158/1940-6207.CAPR-10-0006

[213] Kaefer, C.M. and Milner, J.A. (2011) Herbs and spices in cancer prevention and treatment, in herbal medicine, In: I.F.F. Benzie and S. Wachtel-Galor, Eds., Biomolecular and Clinical Aspects, Llc, Boca Raton.

[214] Gullett, N.P., et al. (2010) Cancer prevention with natural compounds. Seminars in Oncology, 37, 258-281. doi:10.1053/j.seminoncol.2010.06.014

[215] Taylor, C.K., et al. (2009) The effect of genistein aglycone on cancer and cancer risk: A review of in Vitro, preclinical, and clinical studies. Nutrition Reviews, 67, 398415. doi:10.1111/j.1753-4887.2009.00213.x

[216] Gerhauser, C. (2013) Cancer chemoprevention and nutriepigenetics: State of the art and future challenges. Topics in Current Chemistry, 329, 73-132. doi:10.1007/128 $2012 \quad 360$

[217] Li, Y., et al. (2011) Implications of cancer stem cell theory for cancer chemoprevention by natural dietary compounds. The Journal of Nutritional Biochemistry, 22, 799-806. doi:10.1016/j.jnutbio.2010.11.001

[218] Kim, Y.S., et al. (2012) Cancer stem cells: Potential target for bioactive food components. The Journal of Nutritional Biochemistry, 23, 691-698. doi:10.1016/j.jnutbio.2012.03.002

[219] Ullah, M.F., et al. (2012) Ascorbic acid in cancer chemoprevention: Translational perspectives and efficacy. Current Drug Targets, 13, 1757-1771. doi:10.2174/138945012804545669 
[220] Khan, S.I., et al. (2012) Epigenetic events associated with breast cancer and their prevention by dietary components targeting the epigenome. Chemical Research in Toxicology, 25, 61-73. doi:10.1021/tx200378c

[221] Ozten-Kandas, N. and Bosland, M.C. (2011) Chemoprevention of prostate cancer: Natural compounds, antiandrogens, and antioxidants-In Vivo evidence. Journal of Carcinogenesis, 10, 27. doi:10.4103/1477-3163.90438

[222] Khan, N., Adhami, V.M. and Mukhtar, H. (2010) Apoptosis by dietary agents for prevention and treatment of prostate cancer. Endocrine-Related Cancer, 17, R39-R52. doi:10.1677/ERC-09-0262

[223] Howells, L.M., et al. (2011) Phase I randomized, double-blind pilot study of micronized resveratrol (SRT501) in patients with hepatic metastases - Safety, pharmacokinetics, and pharmacodynamics. Cancer Prevention Research, 4, 1419-1425.

doi:10.1158/1940-6207.CAPR-11-0148

[224] Ye, F., et al. (2012) Suppression of esophageal cancer cell growth using curcumin, (-)-epigallocatechin-3-gallate and lovastatin. World Journal of Gastroenterology, 18, 126135. doi:10.3748/wig.v18.i2.126

[225] Hossain, D.M., et al. (2012) Curcumin: The multi-targeted therapy for cancer regression. Front in Bioscience, 4, 335-355.

[226] Basnet, P. and Skalko-Basnet, N. (2011) Curcumin: An anti-inflammatory molecule from a curry spice on the path to cancer treatment. Molecules, 16, 4567-4598. doi:10.3390/molecules 16064567
[227] Darvesh, A.S., Aggarwal, B.B. and Bishayee, A. (2012) Curcumin and liver cancer: A review. Current Pharmaceutical Biotechnology, 13, 218-228.

[228] Shureiqi, I. and Baron, J.A. (2011) Curcumin chemoprevention: The long road to clinical translation. Cancer Prevention Research, 4, 296-298. doi:10.1158/1940-6207.CAPR-11-0060

[229] Rahman, M.A., Amin, A.R. and Shin, D.M. (2010) Chemopreventive potential of natural compounds in head and neck cancer. Nutrition and Cancer, 62, 973-987. doi:10.1080/01635581.2010.509538

[230] Shehzad, A., Wahid, F. and Lee, Y.S. (2010) Curcumin in cancer chemoprevention: Molecular targets, pharmacokinetics, bioavailability, and clinical trials. Archiv der Pharmazie, 343, 489-499. doi:10.1002/ardp.200900319

[231] Juan, M.E., Alfaras, I. and Planas, J.M. (2012) Colorectal cancer chemoprevention by trans-resveratrol. Pharmacological Research, 65, 584-591. doi:10.1016/j.phrs.2012.03.010

[232] Scott, E., et al. (2012) Resveratrol in human cancer chemoprevention-Choosing the "right" dose. Molecular Nutrition \& Food Research, 56, 7-13. doi:10.1002/mnfr.201100400

[233] Vang, O., et al. (2011) What is new for an old molecule? Systematic review and recommendations on the use of resveratrol. PLoS One, 6, e19881. doi:10.1371/journal.pone.0019881 